Supporting Information

\title{
Merobatzelladines A and B, Anti-infective Tricyclic Guanidines from a Marine Sponge Monanchora sp.
}

Shunsuke Takishima, ${ }^{\dagger}$ Aki Ishiyama, ${ }^{\dagger}$ Masahito Iwatsuki, ${ }^{\dagger}$ Kazuhiko Otoguro, ${ }^{\dagger}$ Haruki Yamada, ${ }^{\ddagger}$ Satoshi Ōmura, ${ }^{\ddagger}$ Hirotsugu Kobayashi, ${ }^{\dagger}$ Rob W. M. van Soest, ${ }^{\S}$ and Shigeki Matsunaga $\dagger^{*}$

${ }^{\dagger}$ Laboratory of Aquatic Natural Products Chemistry, Graduate School of Agricultural and Life Science, The University of Tokyo, Bunkyo-ku, Tokyo 113-8657, Japan

${ }^{\ddagger}$ Research Center for Tropical Diseases and Kitasato Institute for Life Sciences, Kitasato University, The Kitasato Institute, 5-9-1 Shirokane, Minato-ku, Tokyo 108-8641, Japan ${ }^{\S}$ Zoological Museum, University of Amsterdam, 1090 GT Amsterdam, The Netherlands

assmats@mail.ec.u-tokyo.ac.jp

\section{Table of contents}

Table of Contents

Experimental Section

Tables S1-S2. Assigned NMR Data for 9-12

3

Figure $\mathrm{S} 1 .{ }^{1} \mathrm{H}$ NMR spectrum of $\mathbf{1}$ in $\mathrm{CD}_{3} \mathrm{OD}$

Figure $\mathrm{S} 2 .{ }^{13} \mathrm{C}$ NMR spectrum of 1 in $\mathrm{CD}_{3} \mathrm{OD}$

8

Figure S3. COSY spectrum of 1 in $\mathrm{CD}_{3} \mathrm{OD}$

9

Figure S4. HSQC spectrum of $\mathbf{1}$ in $\mathrm{CD}_{3} \mathrm{OD}$

Figure S5. HMBC spectrum of $\mathbf{1}$ in $\mathrm{CD}_{3} \mathrm{OD}$

Figure S6. TOCSY spectrum of 1 in $\mathrm{CD}_{3} \mathrm{OD}$

Figure S7. ROESY spectrum of 1 in $\mathrm{CD}_{3} \mathrm{OD}$

Figure S8. ${ }^{1} \mathrm{H}$ NMR spectrum of $\mathbf{2}$ in $\mathrm{CD}_{3} \mathrm{OD}$

Figure S9. ${ }^{13} \mathrm{C}$ NMR spectrum of 2 in $\mathrm{CD}_{3} \mathrm{OD}$ 
$\begin{array}{lr}\text { Figure S11. HSQC spectrum of } \mathbf{2} \text { in } \mathrm{CD}_{3} \mathrm{OD} & 18\end{array}$

Figure S12. HMBC spectrum of 2 in $\mathrm{CD}_{3} \mathrm{OD} \quad 19$

Figure S13. TOCSY spectrum of $\mathbf{2}$ in $\mathrm{CD}_{3} \mathrm{OD} \quad 20$

Figure S14. ROESY spectrum of $\mathbf{2}$ in $\mathrm{CD}_{3} \mathrm{OD} \quad 21$

Figure S15. ${ }^{1} \mathrm{H}$ NMR spectrum of 9 in DMSO- $d_{6}$

Figure S16. COSY spectrum of 9 in DMSO- $d_{6} \quad 23$

Figure S17. HSQC spectrum of 9 in DMSO- $d_{6} \quad 24$

Figure S18. NOESY spectrum of 9 in DMSO- $d_{6} \quad 25$

Figure S19. ${ }^{1} \mathrm{H}$ NMR spectrum of $\mathbf{1 0}$ in DMSO- $d_{6} \quad 26$

$\begin{array}{ll}\text { Figure S20. COSY spectrum of } \mathbf{1 0} \text { in DMSO- } d_{6} & 27\end{array}$

Figure S21. HSQC spectrum of $\mathbf{1 0}$ in DMSO- $d_{6} \quad 28$

Figure S22. NOESY spectrum of $\mathbf{1 0}$ in DMSO- $d_{6} \quad 29$

Figure S23. ${ }^{1} \mathrm{H}$ NMR spectrum of $\mathbf{1 1}$ in $\mathrm{CD}_{3} \mathrm{OD} \quad 30$

Figure S24. COSY spectrum of $\mathbf{1 1}$ in $\mathrm{CD}_{3} \mathrm{OD} \quad 31$

Figure S25. HSQC spectrum of $\mathbf{1 1}$ in $\mathrm{CD}_{3} \mathrm{OD} \quad 32$

Figure S26. NOESY spectrum of $\mathbf{1 1}$ in $\mathrm{CD}_{3} \mathrm{OD}$

Figure S27. ${ }^{1} \mathrm{H}$ NMR spectrum of $\mathbf{1 2}$ in $\mathrm{CD}_{3} \mathrm{OD}$

Figure S28. COSY spectrum of $\mathbf{1 2}$ in $\mathrm{CD}_{3} \mathrm{OD} \quad 35$

Figure S29. HSQC spectrum of $\mathbf{1 2}$ in $\mathrm{CD}_{3} \mathrm{OD} \quad 36$

Figure S30. NOESY spectrum of $\mathbf{1 2}$ in $\mathrm{CD}_{3} \mathrm{OD}$ 


\section{Experimental Section}

For general Experimental Procedures, see Ueoka et al. Biosci. Biotechnol. Biochem., 2008, 72, 3055-3058.

Biological material. The sponge specimen was collected by hand using SCUBA off Amami Oshima $\left(28^{\circ} 8^{\prime} \mathrm{N}, 129^{\circ} 19^{\prime} \mathrm{E}\right)$. The specimen was frozen on site and kept frozen until extraction. The sponge was identified as Monanchora sp. and a voucher specimen (ZMAPOR19862) was deposited at the Zoological Museum of The University of Amsterdam.

Determination of the antimicrobial activity. Antibacterial activity was determined by the paper disk method against fish pathogenic Gram-negative bacteria Vibrio anguillarum (strain: PT-81049 from ayu). The composition of the medium was $1.5 \% \mathrm{NaCl}$, peptone $(15.0 \mathrm{~g})$, soybean peptone $(5.0 \mathrm{~g})$ and agar $(15.0 \mathrm{~g})$ in the $1 \mathrm{~L}$ of $\mathrm{H}_{2} \mathrm{O}$. The samples were prepared above on the paper disk ( $6 \mathrm{~mm}$ of diameter). Diameter of inhibitory zone around the $6 \mathrm{~mm} \phi$ paper disk was measured after incubation for one day at $27^{\circ} \mathrm{C}$.

Determination of anti-parasite acitivity. For the determination of antitrypanosomal and antimalarial activities, see references 15 and 16, respectively.

Extraction and isolation. The sponge (200 g, wet weight) was extracted with $\mathrm{MeOH}$ and a mixture of $\mathrm{CHCl}_{3} / \mathrm{MeOH}(1: 1)$. The extracts were combined, concentrated, and partitioned between water and $\mathrm{CHCl}_{3}$. The $\mathrm{CHCl}_{3}$ layer was further partitioned between $\mathrm{MeOH}-\mathrm{H}_{2} \mathrm{O}$ (9:1) and n-hexane and the resulting aqueous $\mathrm{MeOH}$ layer was diluted with water adjusting the composition of the solvent to $\mathrm{MeOH}-\mathrm{H}_{2} \mathrm{O}(6: 4)$ and extracted with $\mathrm{CHCl}_{3}$. The $\mathrm{CHCl}_{3}$ fraction was separated by ODS flash chromatography with a step-wise elution with mixtures of $\mathrm{MeOH}$ and $\mathrm{H}_{2} \mathrm{O}$. The fraction eluted with $\mathrm{MeOH}-\mathrm{H}_{2} \mathrm{O}$ (7:3) was separated by reversed-phase HPLC (ODS; $\left.\mathrm{H}_{2} \mathrm{O}-\mathrm{MeCN}-\mathrm{TFA}\right)$ to afford Merobatzelladines A (1) and B (2). 
Merobatzelladine A (1): Pale brown oil; $[\alpha]^{25}+37(c$ 0.68, MeOH); UV (MeOH) $\lambda \max 211$ nm (ع 14,000); HRESIMS $m / z 360.3444(\mathrm{M}+\mathrm{H})^{+}$(calcd for $\left.\mathrm{C}_{23} \mathrm{H}_{42} \mathrm{~N}_{3}, \Delta+6.6 \mathrm{mmu}\right) .{ }^{1} \mathrm{H}$ and ${ }^{13} \mathrm{C}$ NMR data, see Table 1.

Merobatzelladine B (2): Pale brown oil; $[\alpha]^{25}+27(c$ 0.15, MeOH); UV (MeOH) $\lambda \max 206$ nm ( $\varepsilon$ 7000); HRESIMS $m / z 306.2909(\mathrm{M}+\mathrm{H}){ }^{+}$(calcd for $\left.\mathrm{C}_{19} \mathrm{H}_{36} \mathrm{~N}_{3}, \Delta+7.0 \mathrm{mmu}\right) .{ }^{1} \mathrm{H}$ and ${ }^{13} \mathrm{C}$ NMR data, see Table 1.

Preparation of 9 and 10 from Merobatzelladine B (2). To a solution of Merobatzelladine B (2) (50 mg) in $3 \mathrm{~mL}$ of dioxane was added $\mathrm{KOH}(31.1 \mathrm{mg})$ and 1,3-ditosyloxy-2-benzyloxypropane $(160.7 \mathrm{mg})$. The reaction mixture was kept at $120{ }^{\circ} \mathrm{C}$ for 1 day, and the reaction mixture was evaporated to dryness. The residue was partitioned between $\mathrm{CHCl}_{3}$ and $\mathrm{H}_{2} \mathrm{O}$ and the $\mathrm{CHCl}_{3}$ layer was purified by ODS HPLC $\left(\mathrm{MeCN}-\mathrm{H}_{2} \mathrm{O}-\mathrm{TFA}\right.$, 7:3:0.01) to afford $8(8 \mathrm{mg})$ and $7(6 \mathrm{mg})$, each of which was subjected to hydrogenation $\left(\mathrm{H}_{2}\right.$, Pd-C, rt, $1 \mathrm{~atm}, 1$ day) to afford $\mathbf{1 0}(4 \mathrm{mg})$ and $\mathbf{9}(5 \mathrm{mg})$, respectively.

9: HRESIMS $m / z 362.3205[\mathrm{M}+\mathrm{H}]^{+}$(calcd for $\left.\mathrm{C}_{22} \mathrm{H}_{40} \mathrm{~N}_{3} \mathrm{O} 362.3171\right),{ }^{1} \mathrm{H}$ and ${ }^{13} \mathrm{C} \mathrm{NMR}$ data see Table S1.

10: HRESIMS m/z $362.3138[\mathrm{M}+\mathrm{H}]^{+}$(calcd for $\mathrm{C}_{22} \mathrm{H}_{40} \mathrm{~N}_{3} \mathrm{O} 362.3171$ ), ${ }^{1} \mathrm{H}$ and ${ }^{13} \mathrm{C}$ NMR data see Table S1.

MTPA esterification. To a solution of $\mathbf{1 0}(1 \mathrm{mg})$ in pyridine $(50 \mu \mathrm{L})$ was added $(S)$-MTPACl $(6 \mu \mathrm{L})$. The reaction mixture was stirred at $\mathrm{rt}$ for $30 \mathrm{~min}$, and the solvent was evaporated. The residue was partitioned between $1 \mathrm{M} \mathrm{NaHCO}_{3}$ and $\mathrm{CHCl}_{3}$ and the $\mathrm{CHCl}_{3}$ fraction was purified by ODS HPLC (MeCN-H $\left.{ }_{2} \mathrm{O}-\mathrm{TFA}, 7: 3: 0.01\right)$ to yield the (R)-MTPA ester $11(0.8 \mathrm{mg})$. The $(S)$-MTPA ester 12 was prepared in the same way.

11: HRESIMS $m / z 578.3570[\mathrm{M}+\mathrm{H}]^{+}$(calcd for $\mathrm{C}_{32} \mathrm{H}_{47} \mathrm{~N}_{3} \mathrm{~F}_{3} \mathrm{O}_{3} 578.3570$ ), ${ }^{1} \mathrm{H}$ and ${ }^{13} \mathrm{C} \mathrm{NMR}$ data see Table S2. 
12: HRESIMS $m / z 578.3611[\mathrm{M}+\mathrm{H}]^{+}$(calcd for $\mathrm{C}_{32} \mathrm{H}_{47} \mathrm{~N}_{3} \mathrm{~F}_{3} \mathrm{O}_{3} 578.3570$ ), ${ }^{1} \mathrm{H}$ and ${ }^{13} \mathrm{C} \mathrm{NMR}$ data see Table S2. 
Table S1. ${ }^{1} \mathrm{H}$ and ${ }^{13} \mathrm{C}$ NMR Data for 9 and 10 in DMSO- $d_{6}$

\begin{tabular}{|c|c|c|c|c|}
\hline & \multicolumn{2}{|c|}{9} & \multicolumn{2}{|c|}{10} \\
\hline position & $\delta_{\mathrm{H}}($ mult,$J \mathrm{~Hz})$ & $\delta_{C}\left(\right.$ mult $\left.^{\mathrm{b}}\right)$ & $\delta_{\mathrm{H}}(\mathrm{mult}, J \mathrm{~Hz})$ & $\delta_{\mathrm{C}}\left(\right.$ mult $\left.^{\mathrm{b}}\right)$ \\
\hline 1'a & $1.43(\mathrm{~m})$ & $32.4\left(\mathrm{CH}_{2}\right)$ & $1.45(\mathrm{~m})$ & $30.7\left(\mathrm{CH}_{2}\right)$ \\
\hline 1 'b & $1.77(\mathrm{~m})$ & & $1.77(\mathrm{~m})$ & \\
\hline 1 & $3.35(\mathrm{~m})$ & $55.7(\mathrm{CH})$ & $3.40(\mathrm{~m})$ & $55.3(\mathrm{CH})$ \\
\hline $2 \mathrm{a}$ & $1.42(\mathrm{~m})$ & $32.4\left(\mathrm{CH}_{2}\right)$ & $1.43(\mathrm{~m})$ & $32.4\left(\mathrm{CH}_{2}\right)$ \\
\hline $2 b$ & $2.17(\mathrm{~m})$ & & $2.18(\mathrm{~m})$ & \\
\hline 3 & $3.67(\mathrm{~m})$ & $52.9(\mathrm{CH})$ & $3.66(\mathrm{~m})$ & $53.3(\mathrm{CH})$ \\
\hline $4 a$ & $1.73(\mathrm{~m})$ & $29.1\left(\mathrm{CH}_{2}\right)$ & $1.71(\mathrm{~m})$ & $29.0\left(\mathrm{CH}_{2}\right)$ \\
\hline $4 b$ & $2.14(\mathrm{~m})$ & & $2.14(\mathrm{~m})$ & \\
\hline $5 \mathrm{a}$ & $1.45(\mathrm{~m})$ & $29.3\left(\mathrm{CH}_{2}\right)$ & $1.45(\mathrm{~m})$ & $29.2\left(\mathrm{CH}_{2}\right)$ \\
\hline $5 b$ & $2.04(\mathrm{~m})$ & & $2.04(\mathrm{~m})$ & \\
\hline 6 & $3.64(\mathrm{~m})$ & $53.9(\mathrm{CH})$ & $3.63(\mathrm{~m})$ & $53.9(\mathrm{CH})$ \\
\hline $7 \mathrm{a}$ & $1.53(\mathrm{~m})$ & $27.3\left(\mathrm{CH}_{2}\right)$ & $1.53(\mathrm{~m})$ & $27.7\left(\mathrm{CH}_{2}\right)$ \\
\hline $7 b$ & $2.23(\mathrm{dd})$ & & $2.17(\mathrm{dd})$ & \\
\hline 8 & $3.36(\mathrm{~m})$ & $56.4(\mathrm{CH})$ & $3.43(\mathrm{~m})$ & $57.1(\mathrm{CH})$ \\
\hline \multicolumn{5}{|l|}{9} \\
\hline $10 \mathrm{a}$ & $3.04(\mathrm{~m})$ & $50.4\left(\mathrm{CH}_{2}\right)$ & $3.05(\mathrm{~m})$ & $49.0\left(\mathrm{CH}_{2}\right)$ \\
\hline $10 \mathrm{~b}$ & $3.41(\mathrm{dd})$ & & $3.27(\mathrm{dd})$ & \\
\hline 11 & $4.16(\mathrm{~m})$ & $58.7(\mathrm{CH})$ & $3.94(\mathrm{~m})$ & $60.3(\mathrm{CH})$ \\
\hline $12 \mathrm{a}$ & $3.24(\mathrm{~m})$ & $50.1\left(\mathrm{CH}_{2}\right)$ & $3.08(\mathrm{~m})$ & $51.3\left(\mathrm{CH}_{2}\right)$ \\
\hline $12 b$ & $3.39(\mathrm{dd})$ & & $3.48(\mathrm{dd})$ & \\
\hline $11-\mathrm{OH}$ & $5.40(\mathrm{~s})$ & & $5.54(\mathrm{~s})$ & \\
\hline $1 " \mathrm{a}$ & $1.40(\mathrm{~m})$ & $30.7\left(\mathrm{CH}_{2}\right)$ & $1.41(\mathrm{~m})$ & $30.8\left(\mathrm{CH}_{2}\right)$ \\
\hline $1 " \mathrm{~b}$ & $1.69(\mathrm{~m})$ & & $1.59(\mathrm{~m})$ & \\
\hline
\end{tabular}


Table S2. ${ }^{1} \mathrm{H}$ and ${ }^{13} \mathrm{C}$ NMR Data for $\mathbf{1 1}$ and $\mathbf{1 2}$ in $\mathrm{CD}_{3} \mathrm{OD}$

\begin{tabular}{|c|c|c|c|c|c|}
\hline \multicolumn{3}{|c|}{11} & \multicolumn{2}{|c|}{12} & \multirow[t]{2}{*}{$\Delta \delta$} \\
\hline position & $\delta_{\mathrm{H}}($ mult,$J \mathrm{~Hz})$ & $\delta_{\mathrm{C}}\left(\mathrm{mult}^{\mathrm{b}}\right)$ & $\delta_{\mathrm{H}}($ mult,$J \mathrm{~Hz})$ & $\delta_{\mathrm{C}}\left(\right.$ mult $\left.^{\mathrm{b}}\right)$ & \\
\hline 1'a & $1.25(\mathrm{~m})$ & $33.0\left(\mathrm{CH}_{2}\right)$ & $1.24(\mathrm{~m})$ & $33.5\left(\mathrm{CH}_{2}\right)$ & -0.01 \\
\hline $1^{\prime} \mathrm{b}$ & $1.69(\mathrm{~m})$ & & $1.83(\mathrm{~m})$ & & +0.14 \\
\hline 1 & $3.41(\mathrm{~m})$ & $57.2(\mathrm{CH})$ & $3.39(\mathrm{~m})$ & $56.9(\mathrm{CH})$ & -0.02 \\
\hline $2 a$ & $1.30(\mathrm{~m})$ & $34.8\left(\mathrm{CH}_{2}\right)$ & $1.15(\mathrm{~m})$ & $34.6\left(\mathrm{CH}_{2}\right)$ & -0.15 \\
\hline $2 b$ & $2.30(\mathrm{~m})$ & & $2.29(\mathrm{~m})$ & & -0.01 \\
\hline 3 & $3.74(\mathrm{~m})$ & $56.1(\mathrm{CH})$ & $3.70(\mathrm{~m})$ & $55.7(\mathrm{CH})$ & -0.04 \\
\hline $4 a$ & $1.73(\mathrm{~m})$ & $30.6\left(\mathrm{CH}_{2}\right)$ & $1.73(\mathrm{~m})$ & $30.8\left(\mathrm{CH}_{2}\right)$ & 0 \\
\hline $4 \mathrm{~b}$ & $2.23(\mathrm{~m})$ & & $2.24(\mathrm{~m})$ & & +0.01 \\
\hline $5 a$ & $1.53(\mathrm{~m})$ & $30.9\left(\mathrm{CH}_{2}\right)$ & $1.40(\mathrm{~m})$ & $30.7\left(\mathrm{CH}_{2}\right)$ & -0.13 \\
\hline $5 b$ & $2.17(\mathrm{~m})$ & & $2.13(\mathrm{~m})$ & & -0.04 \\
\hline 6 & $3.76(\mathrm{~m})$ & $55.4(\mathrm{CH})$ & $3.70(\mathrm{~m})$ & $55.7(\mathrm{CH})$ & -0.06 \\
\hline $7 \mathrm{a}$ & $1.50(\mathrm{~m})$ & $29.5\left(\mathrm{CH}_{2}\right)$ & $1.34(\mathrm{~m})$ & $29.5\left(\mathrm{CH}_{2}\right)$ & -0.16 \\
\hline $7 b$ & $2.30(\mathrm{dd})$ & & $2.24(\mathrm{dd})$ & & -0.06 \\
\hline 8 & $3.44(\mathrm{~m})$ & $60.0(\mathrm{CH})$ & $3.34(\mathrm{~m})$ & $59.8(\mathrm{CH})$ & -0.10 \\
\hline \multicolumn{6}{|l|}{9} \\
\hline $10 \mathrm{a}$ & $3.49(\mathrm{~m})$ & $45.9\left(\mathrm{CH}_{2}\right)$ & $3.47(\mathrm{~m})$ & $45.2\left(\mathrm{CH}_{2}\right)$ & -0.02 \\
\hline $10 \mathrm{~b}$ & $3.53(\mathrm{dd})$ & & $3.74(\mathrm{dd})$ & & +0.19 \\
\hline 11 & $5.54(\mathrm{~m})$ & $67.8(\mathrm{CH})$ & $5.51(\mathrm{~m})$ & $67.9(\mathrm{CH})$ & -0.03 \\
\hline $12 \mathrm{a}$ & $3.50(\mathrm{~m})$ & $50.6\left(\mathrm{CH}_{2}\right)$ & $3.43(\mathrm{~m})$ & $50.9\left(\mathrm{CH}_{2}\right)$ & -0.07 \\
\hline 12 & $3.95(\mathrm{dd})$ & & $3.93(\mathrm{dd})$ & & -0.02 \\
\hline $1 \mathrm{la}$ & $1.56(\mathrm{~m})$ & $32.5\left(\mathrm{CH}_{2}\right)$ & $1.54(\mathrm{~m})$ & $32.2\left(\mathrm{CH}_{2}\right)$ & -0.02 \\
\hline $1 " \mathrm{~b}$ & $1.79(\mathrm{~m})$ & & $1.76(\mathrm{~m})$ & & -0.03 \\
\hline
\end{tabular}




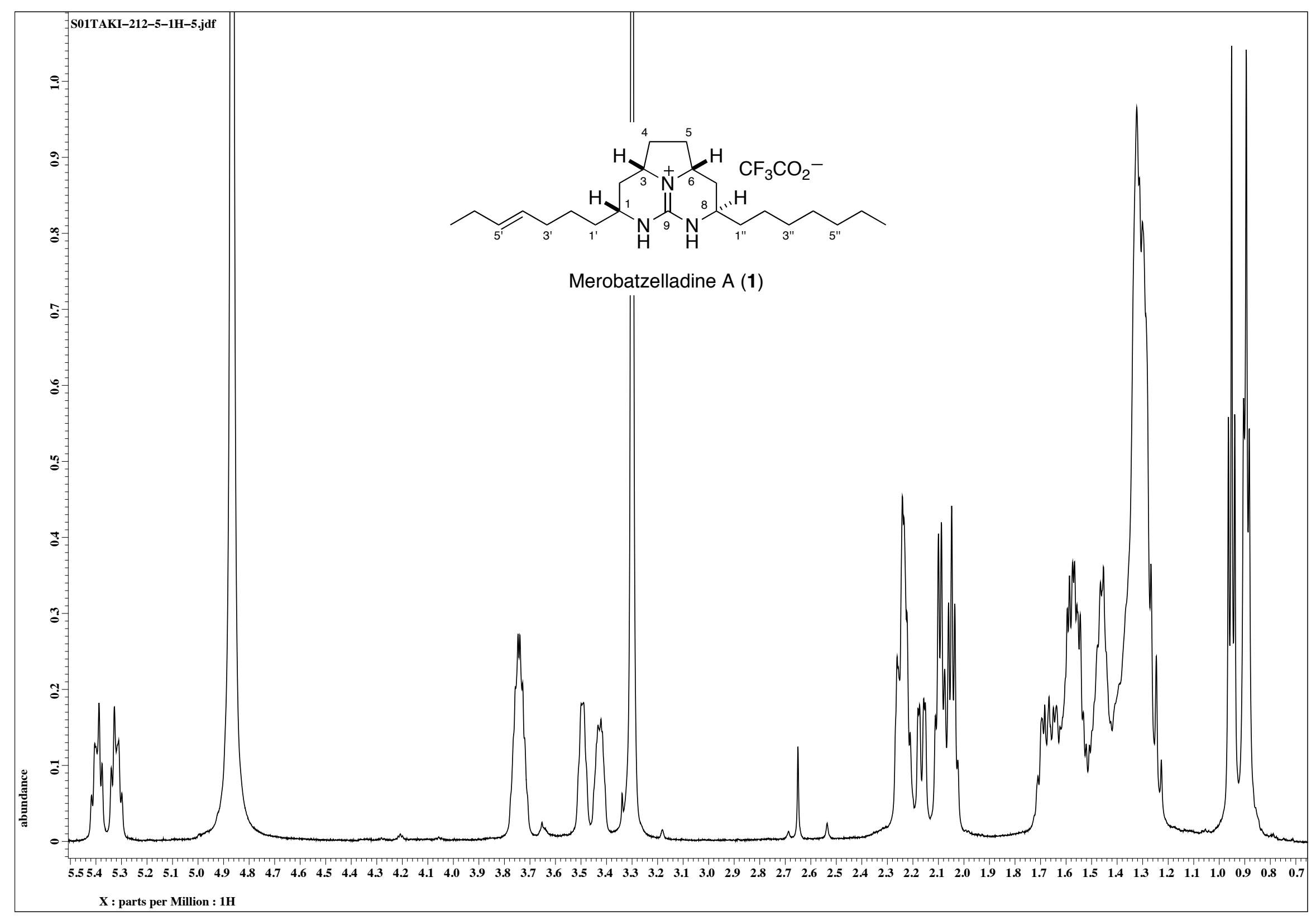

Figure S1. ${ }^{1} \mathrm{H}$ NMR spectrum of $\mathbf{1}$ in $\mathrm{CD}_{3} \mathrm{OD}$ 


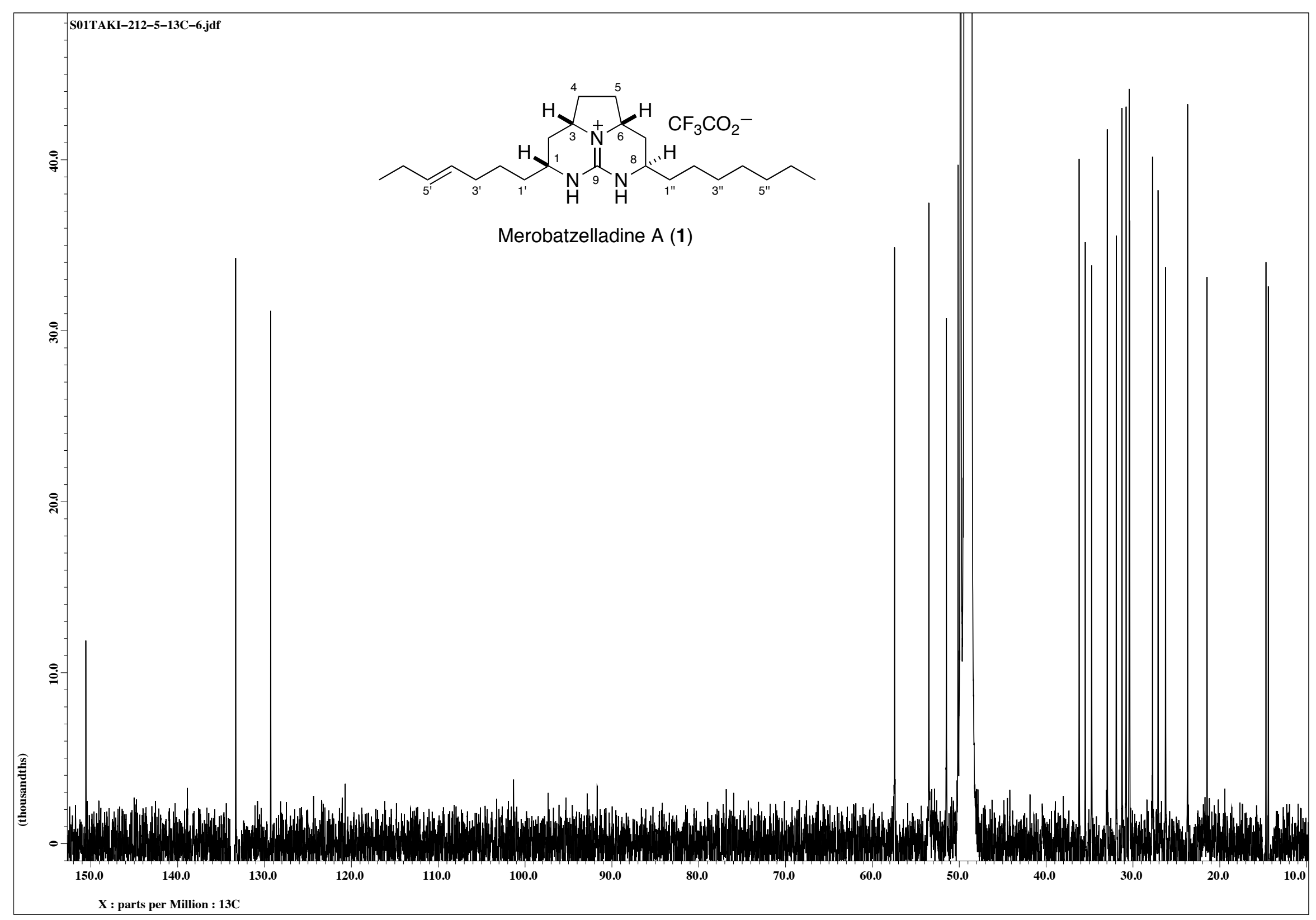

Figure S2. ${ }^{13} \mathrm{C}$ NMR spectrum of $\mathbf{1}$ in $\mathrm{CD}_{3} \mathrm{OD}$ 


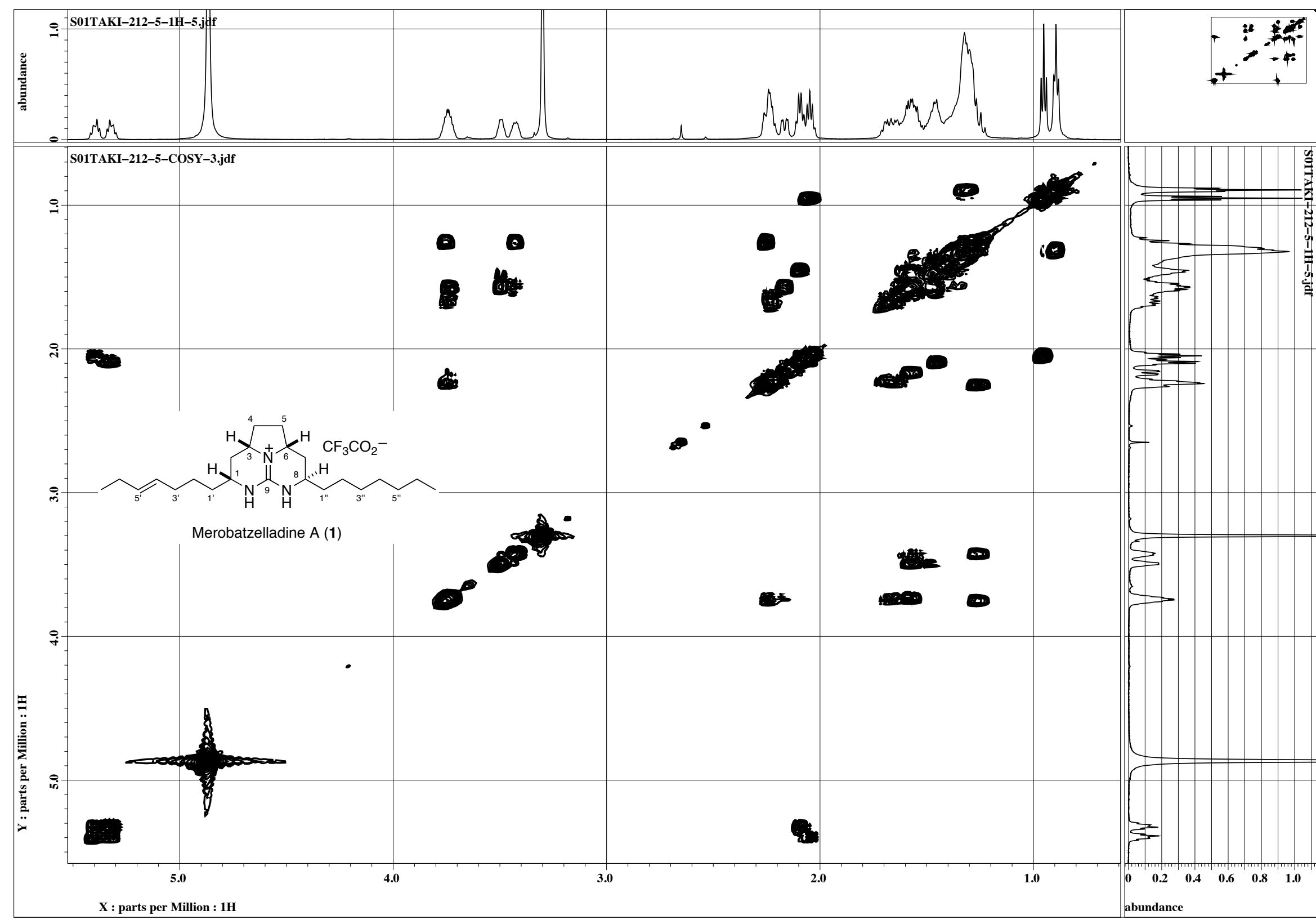

Figure S3. COSY spectrum of $\mathbf{1}$ in $\mathrm{CD}_{3} \mathrm{OD}$ 


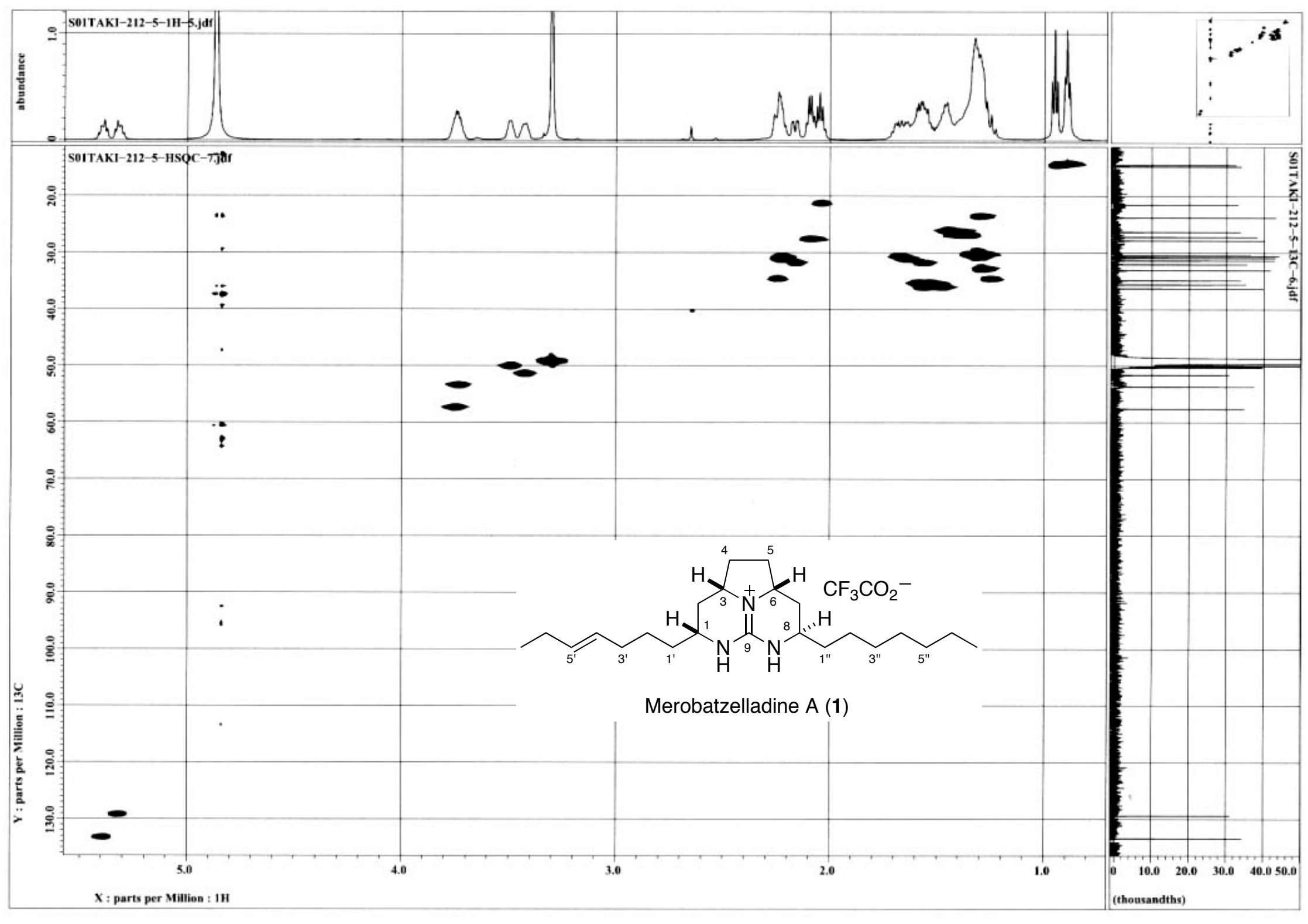

Figure S4. HSQC spectrum of $\mathbf{1}$ in $\mathrm{CD}_{3} \mathrm{OD}$

11 


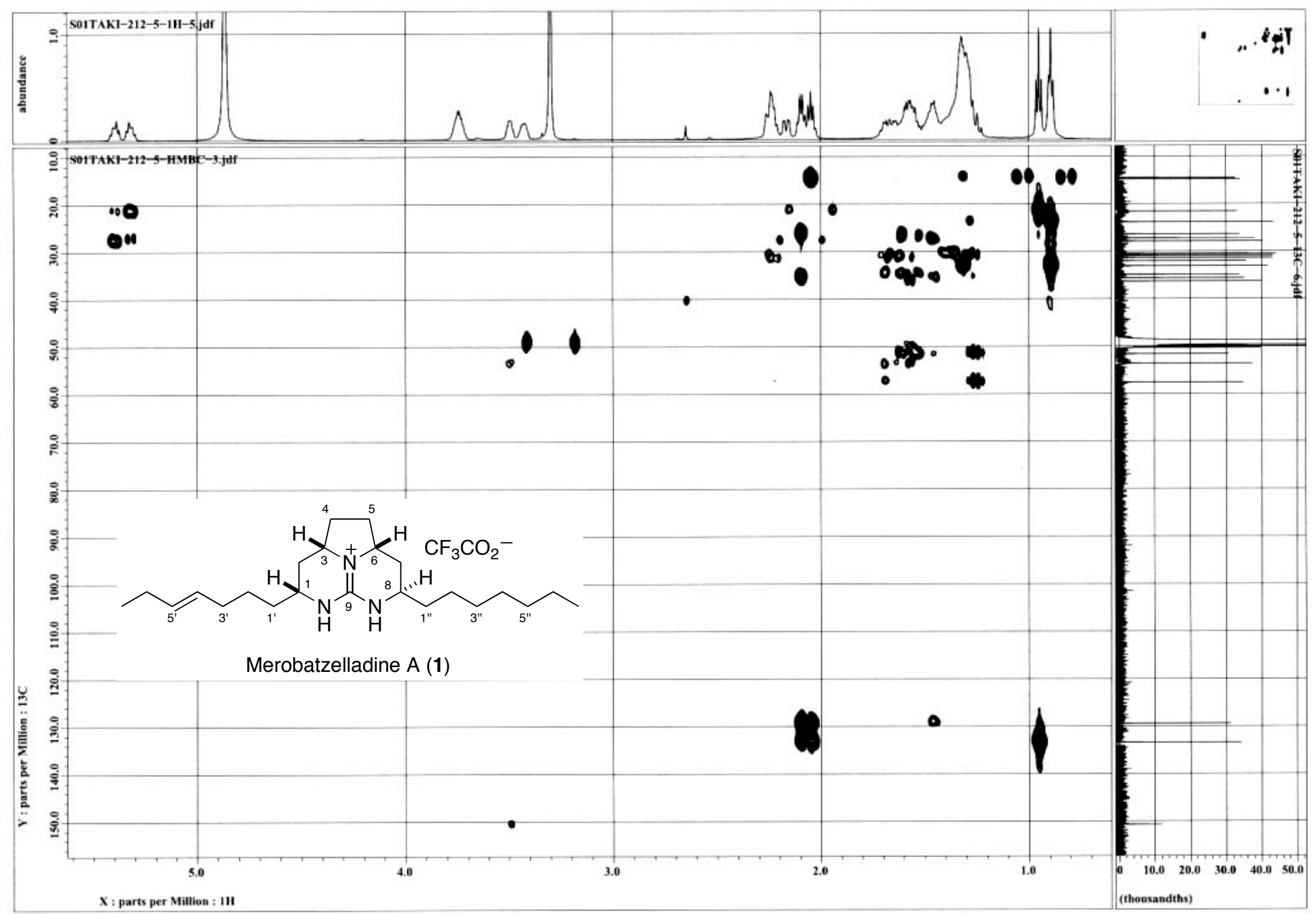

Figure S5. $\mathrm{HMBC}$ spectrum of $\mathbf{1}$ in $\mathrm{CD}_{3} \mathrm{OD}$ 


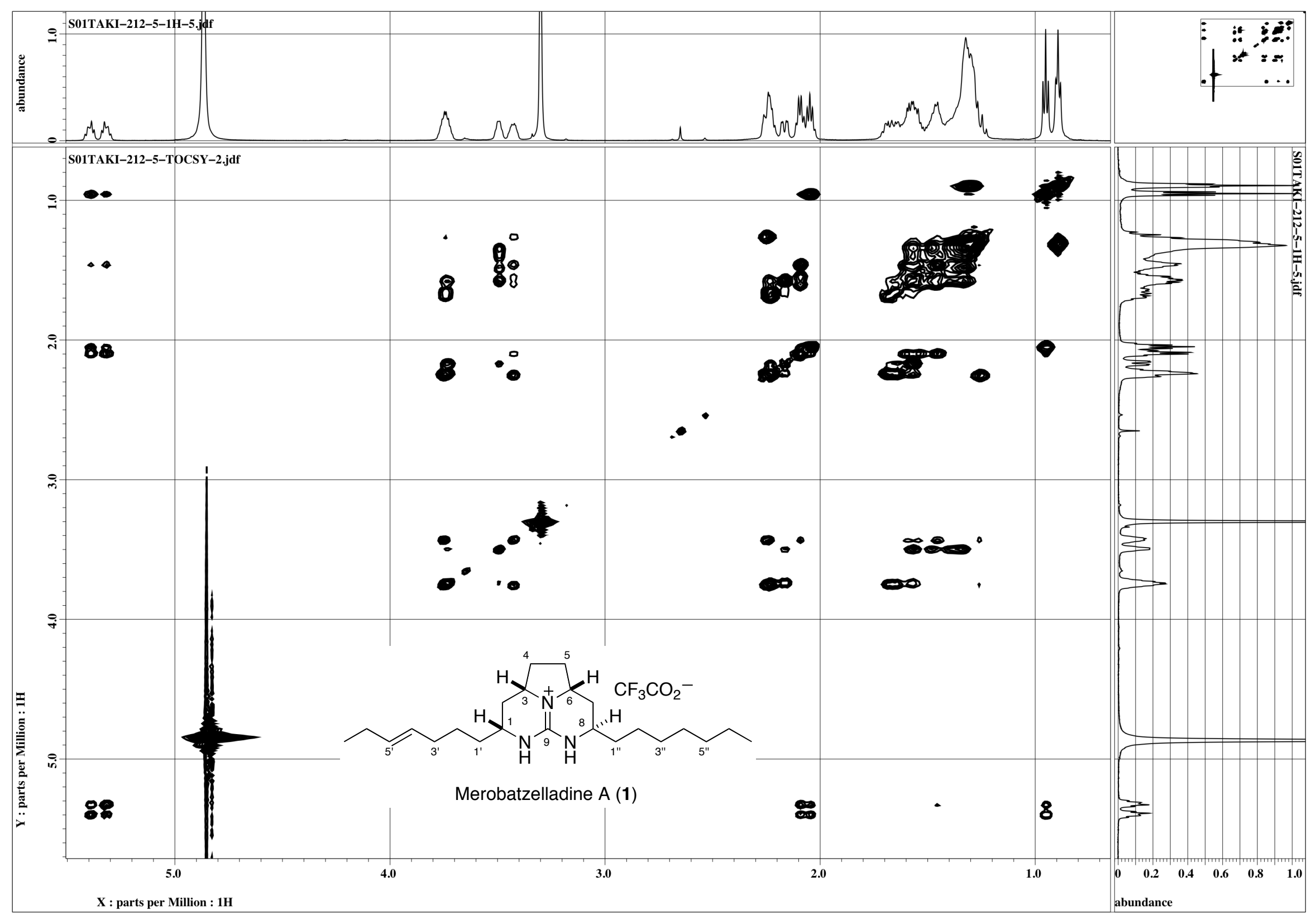

Figure S6. TOCSY spectrum of 1 in $\mathrm{CD}_{3} \mathrm{OD}$ 


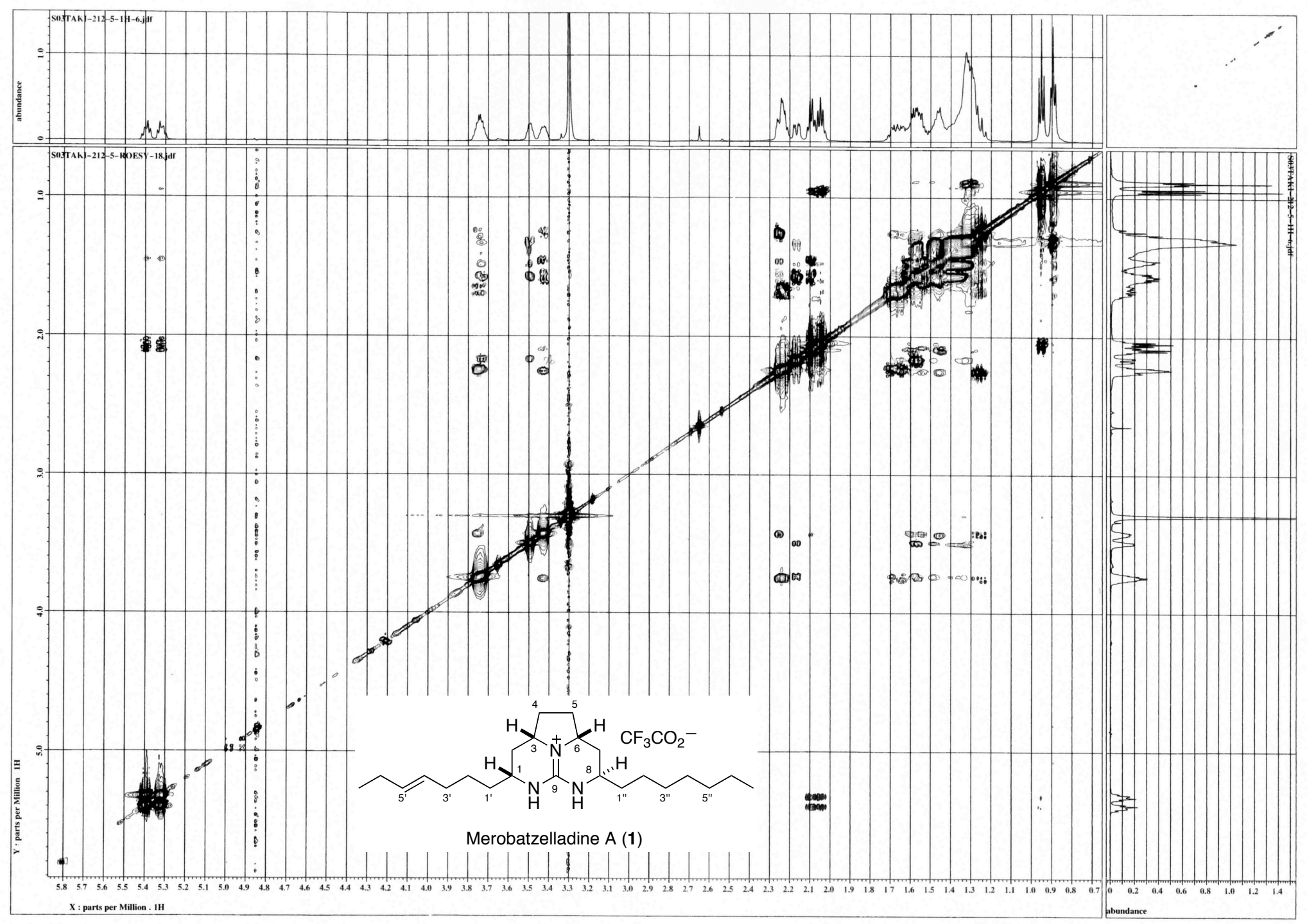

Figure S7. ROESY spectrum of $\mathbf{1}$ in $\mathrm{CD}_{3} \mathrm{OD}$ 


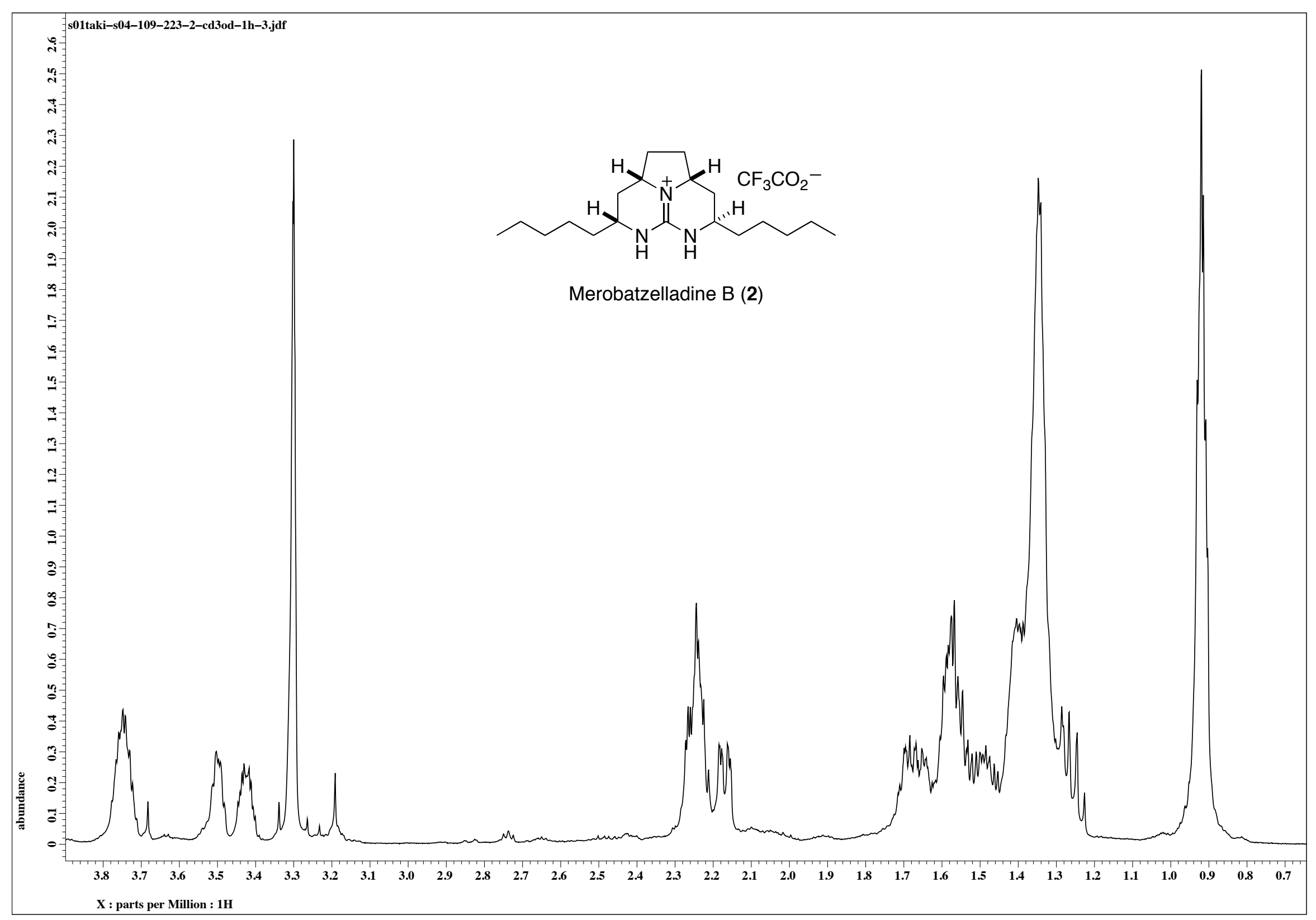

Figure S8. ${ }^{1} \mathrm{H}$ NMR spectrum of $\mathbf{2}$ in $\mathrm{CD}_{3} \mathrm{OD}$ 


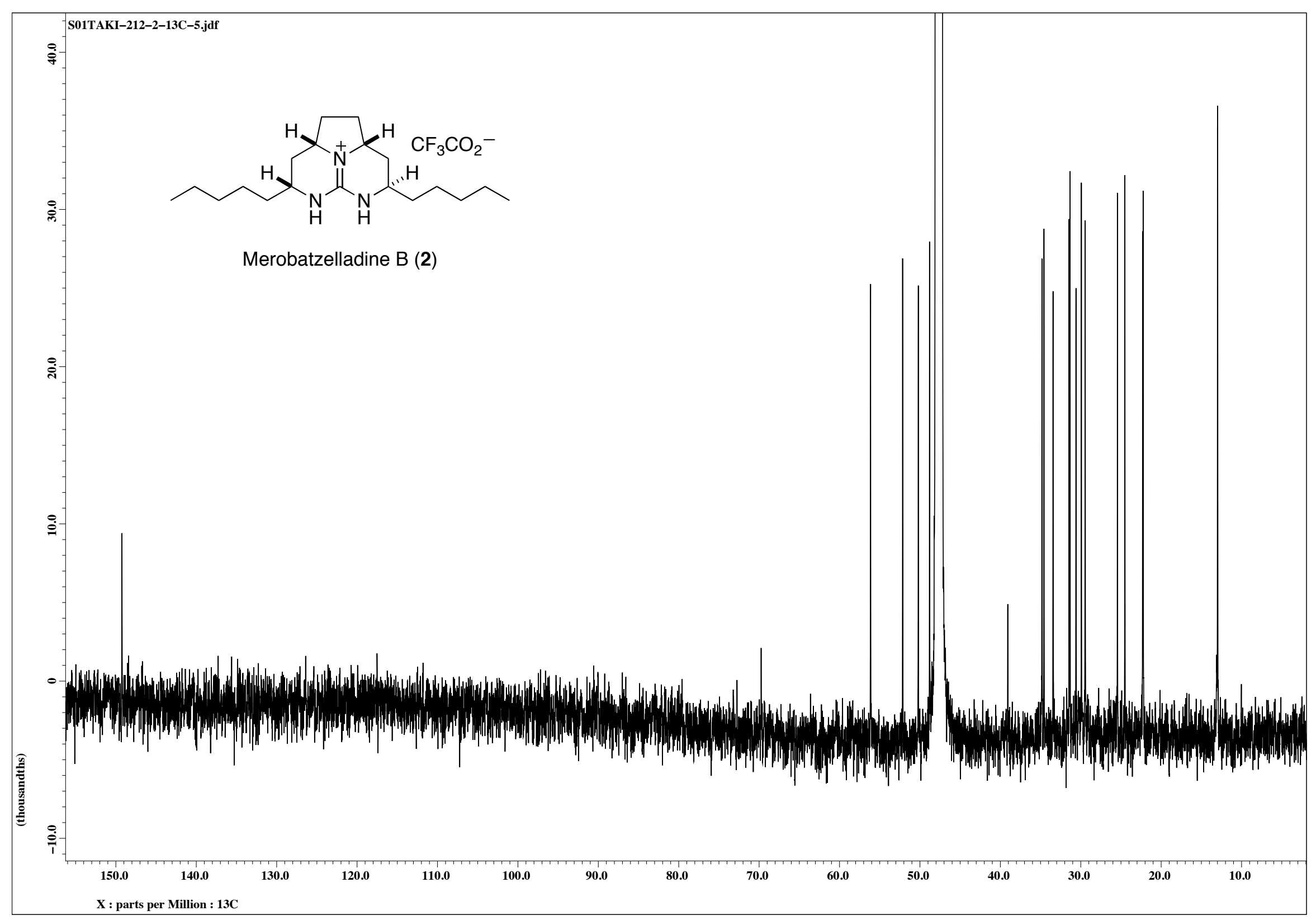

Figure S9. ${ }^{13} \mathrm{C}$ NMR spectrum of $\mathbf{2}$ in $\mathrm{CD}_{3} \mathrm{OD}$ 


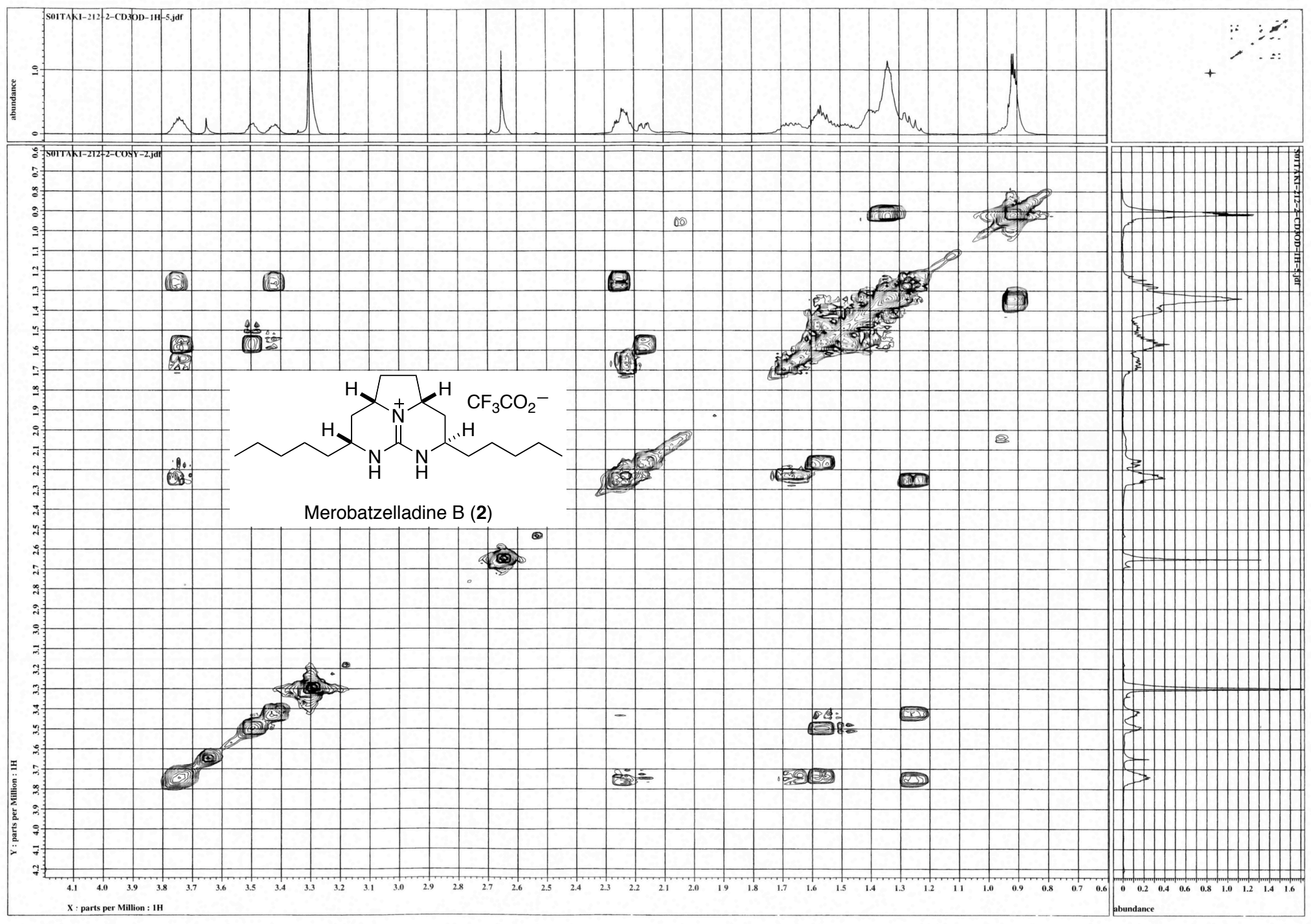

Figure S10. COSY spectrum of 2 in $\mathrm{CD}_{3} \mathrm{OD}$ 


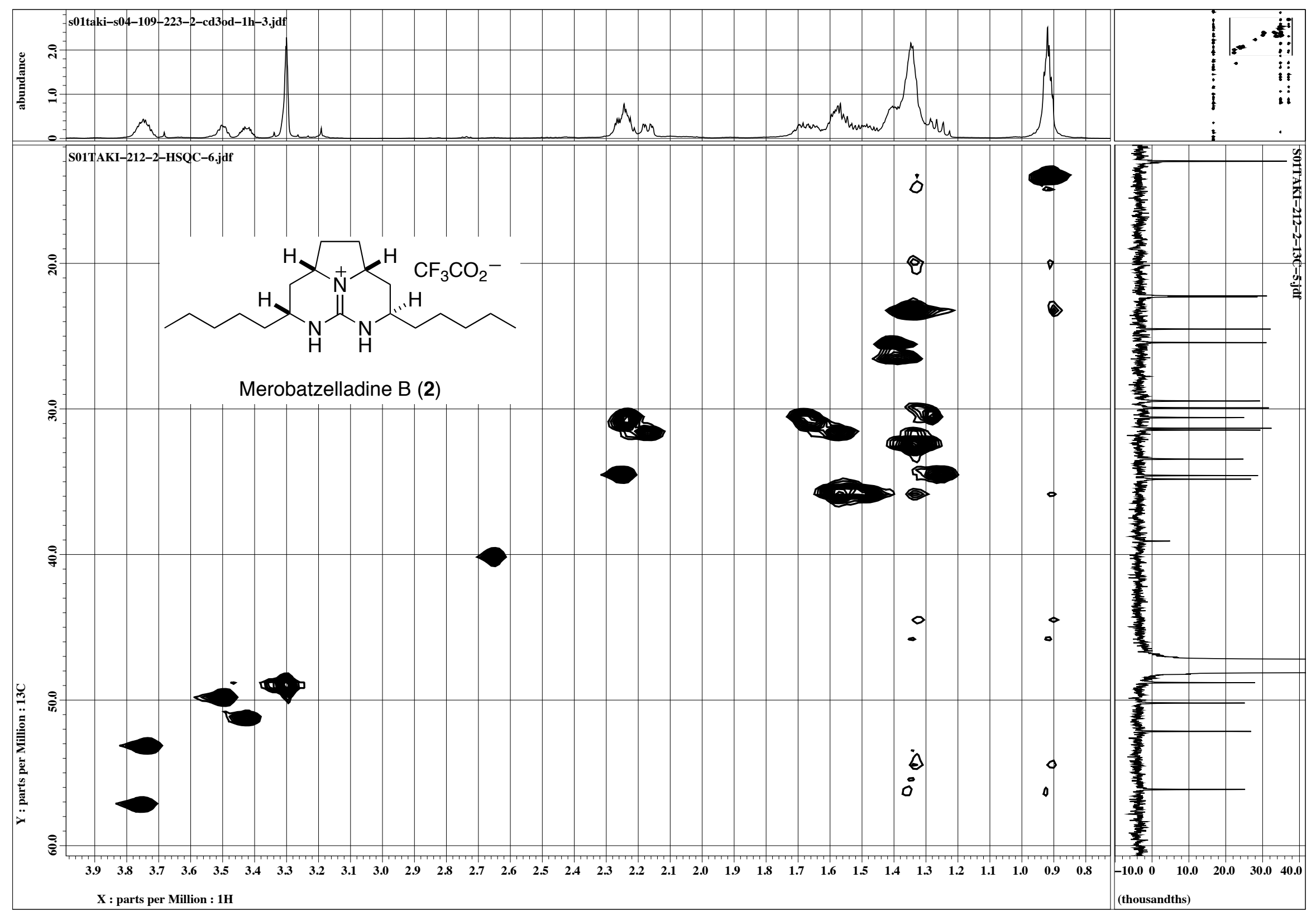

Figure S11. HSQC spectrum of 2 in $\mathrm{CD}_{3} \mathrm{OD}$ 


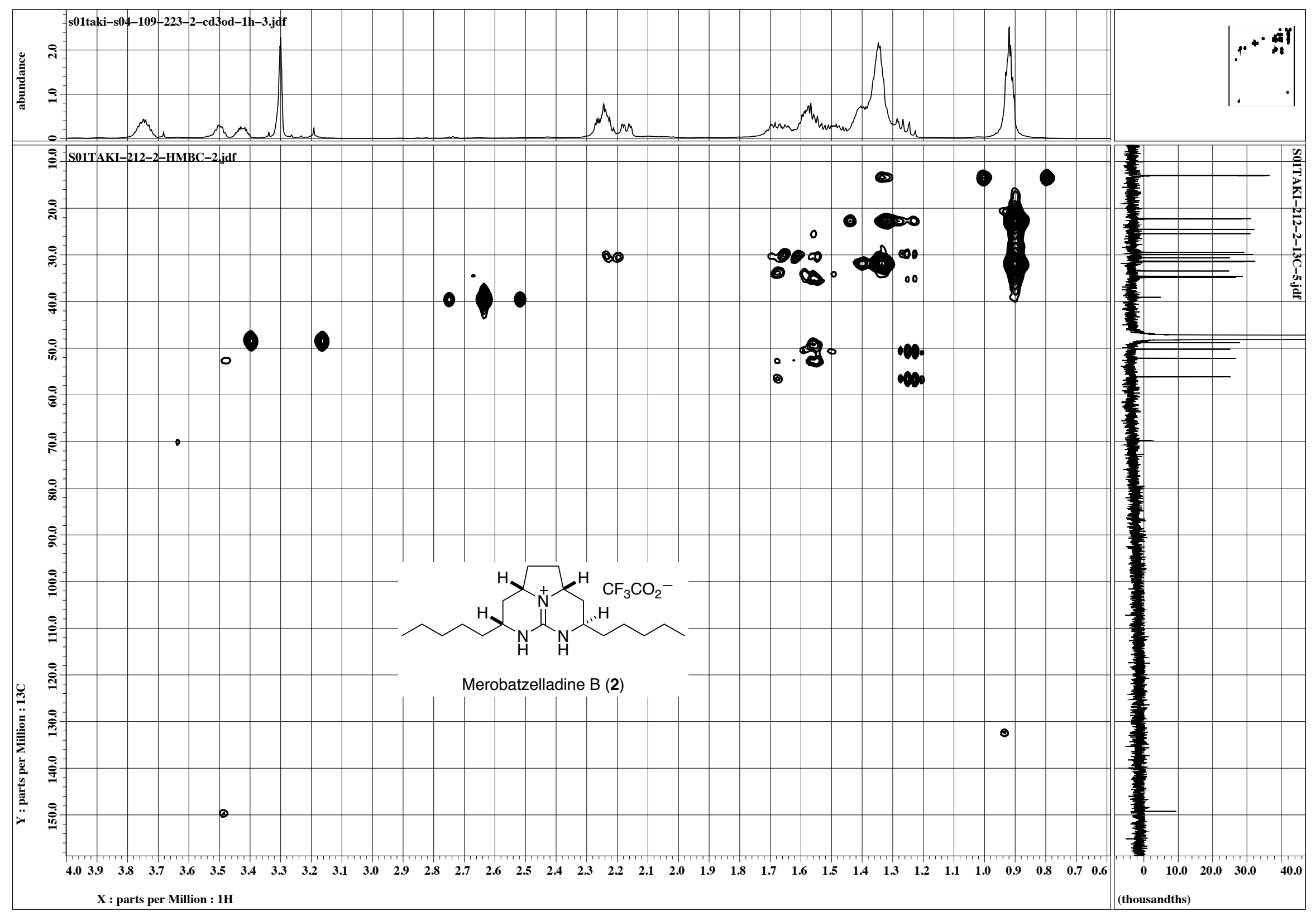

Figure S12. HMBC spectrum of 2 in $\mathrm{CD}_{3} \mathrm{OD}$ 


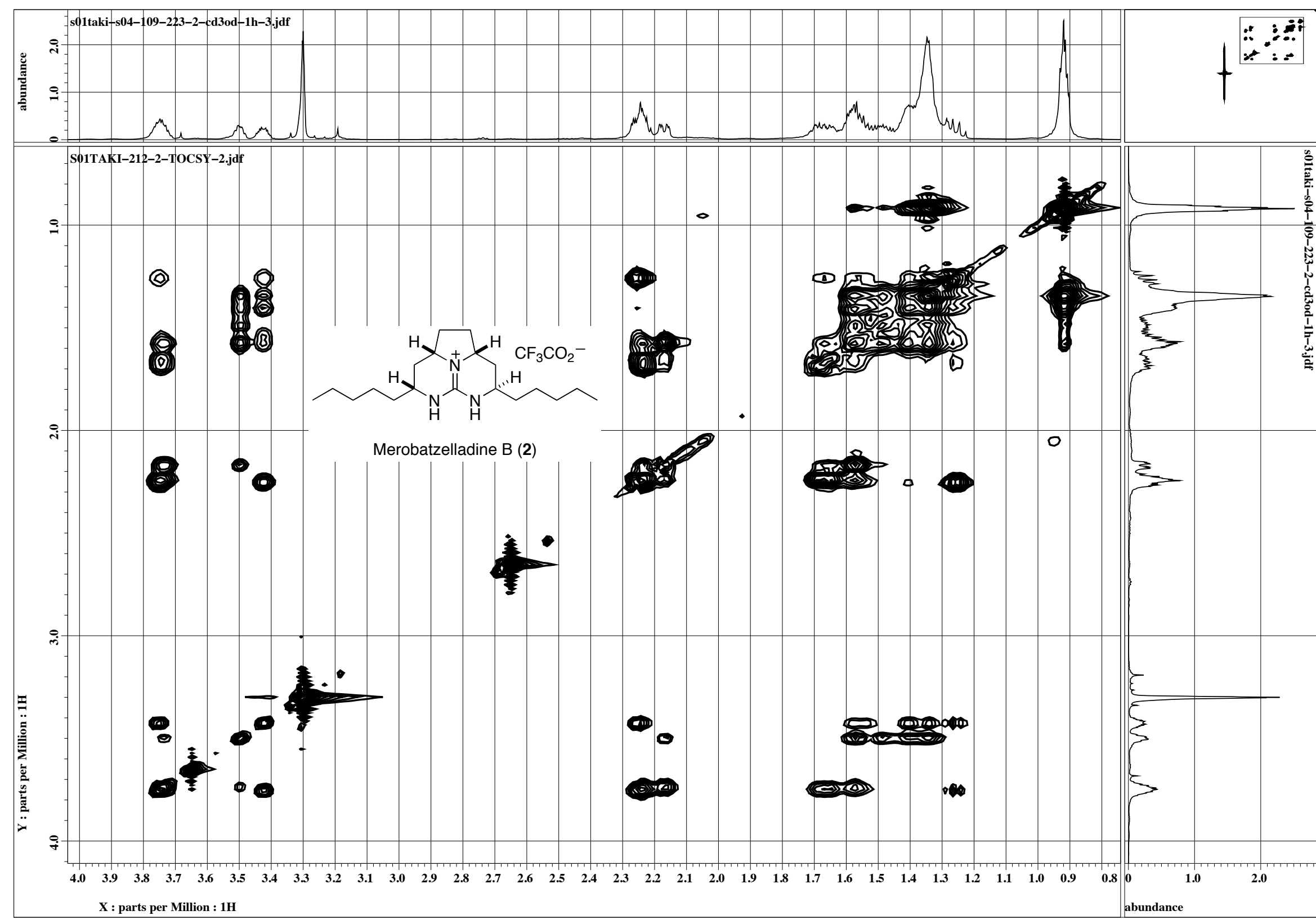

Figure S13. TOCSY spectrum of $\mathbf{2}$ in $\mathrm{CD}_{3} \mathrm{OD}$ 


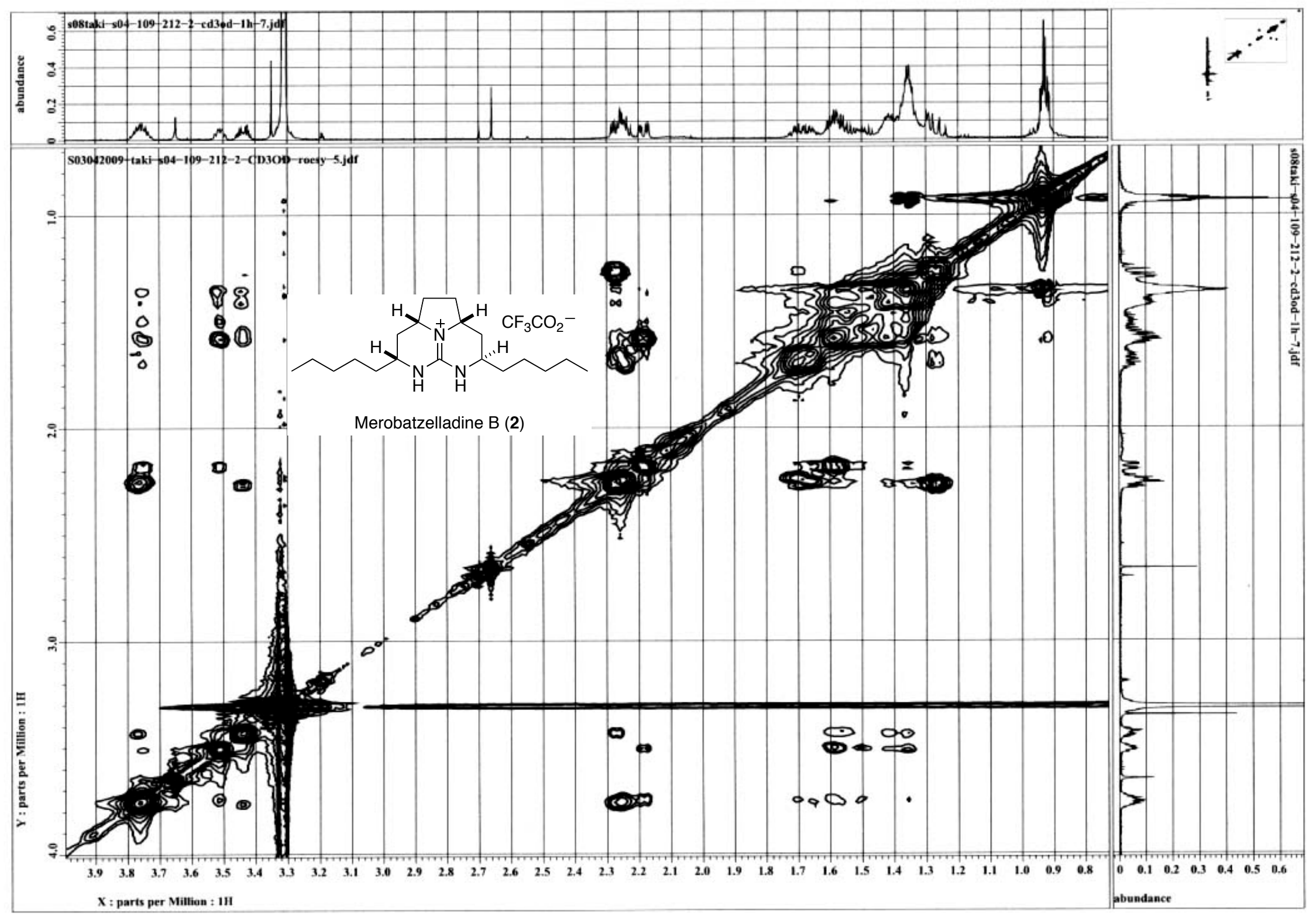

Figure S14. ROESY spectrum of $\mathbf{2}$ in $\mathrm{CD}_{3} \mathrm{OD}$ 


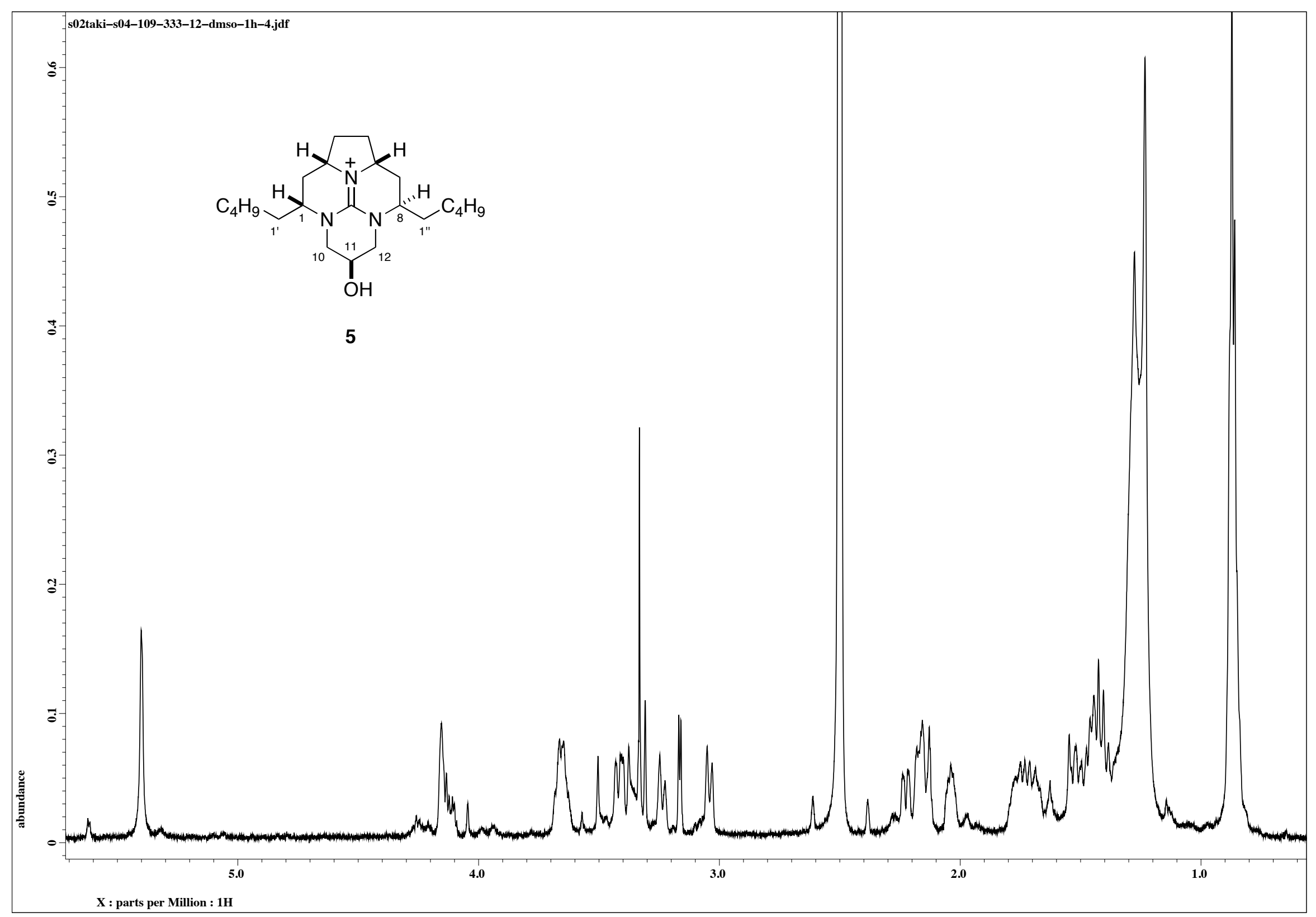

Figure S15. ${ }^{1} \mathrm{H}$ NMR spectrum of 5 in DMSO- $d_{6}$ 


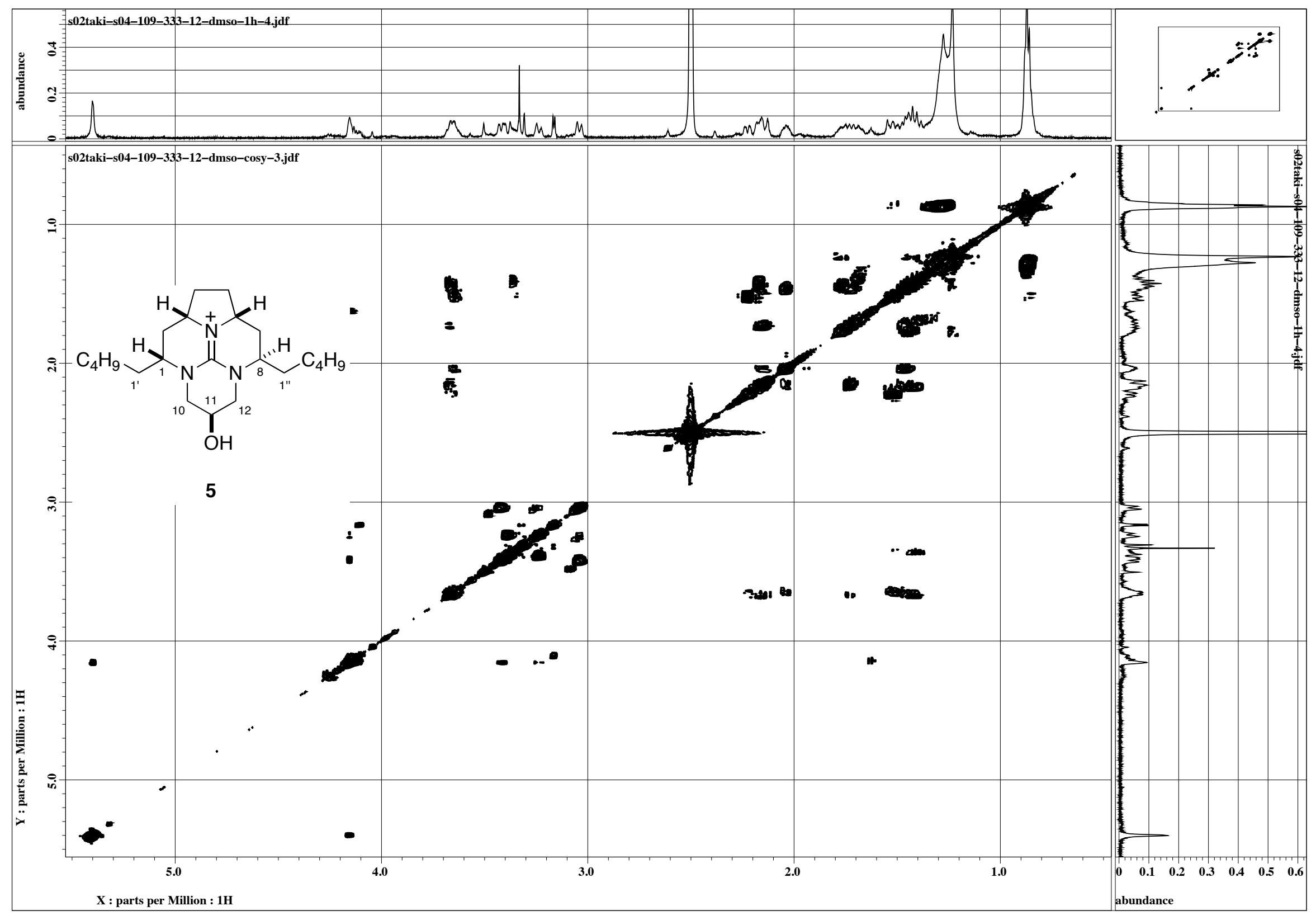

Figure S16. COSY spectrum of 5 in DMSO- $d_{6}$ 


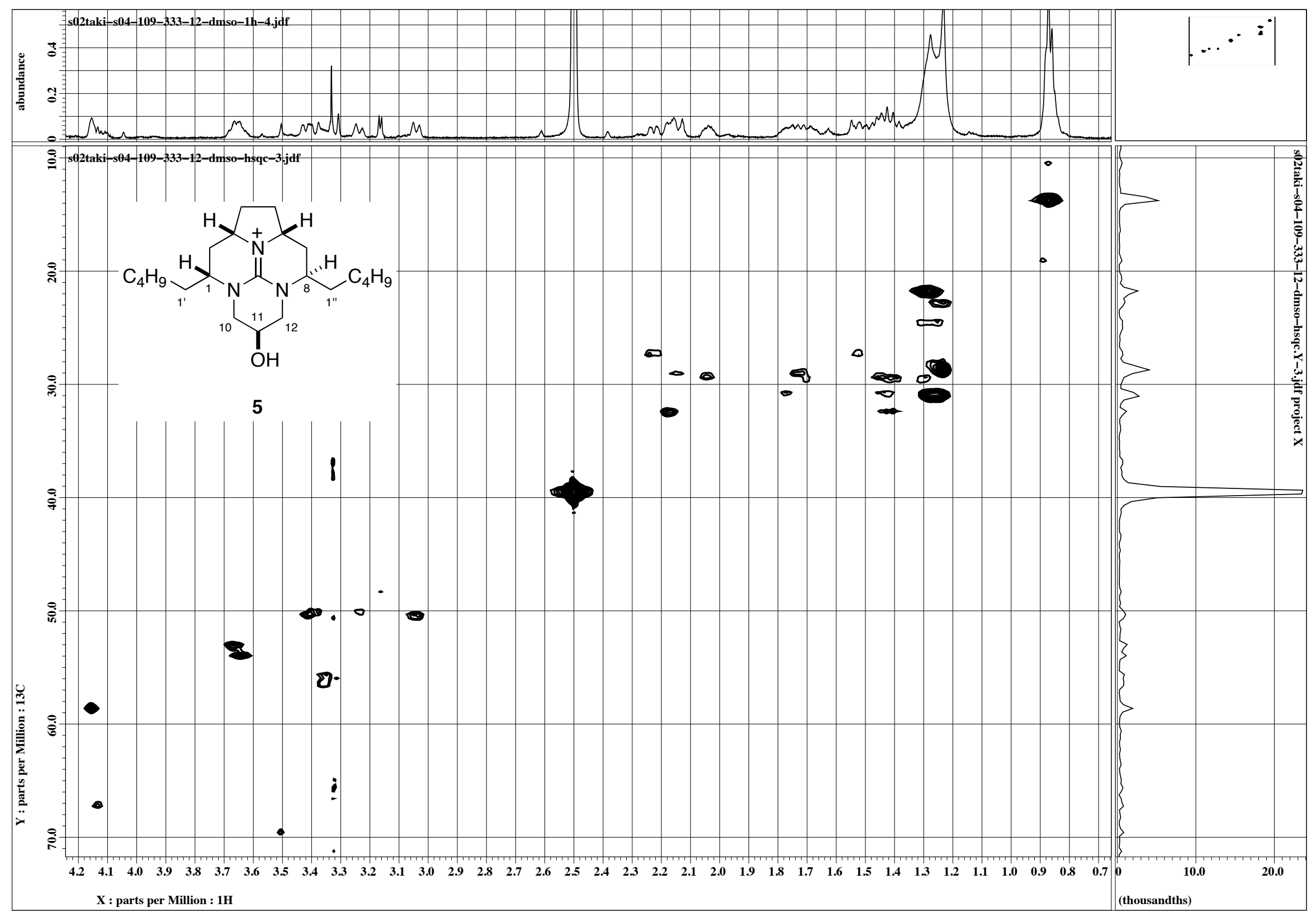

Figure S17. HSQC spectrum of 5 in DMSO- $d_{6}$ 


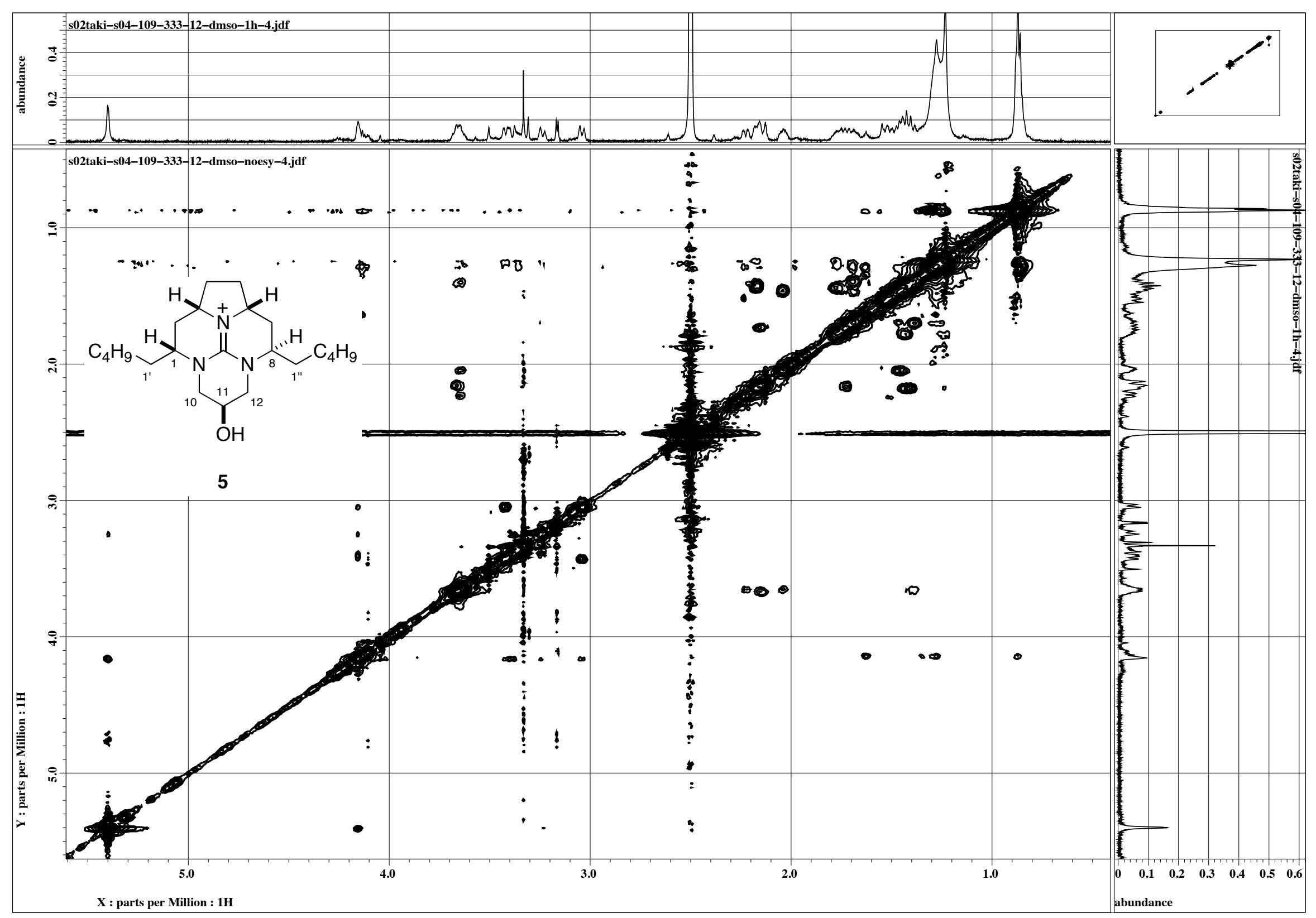

Figure S18. NOESY spectrum of 5 in DMSO- $d_{6}$ 


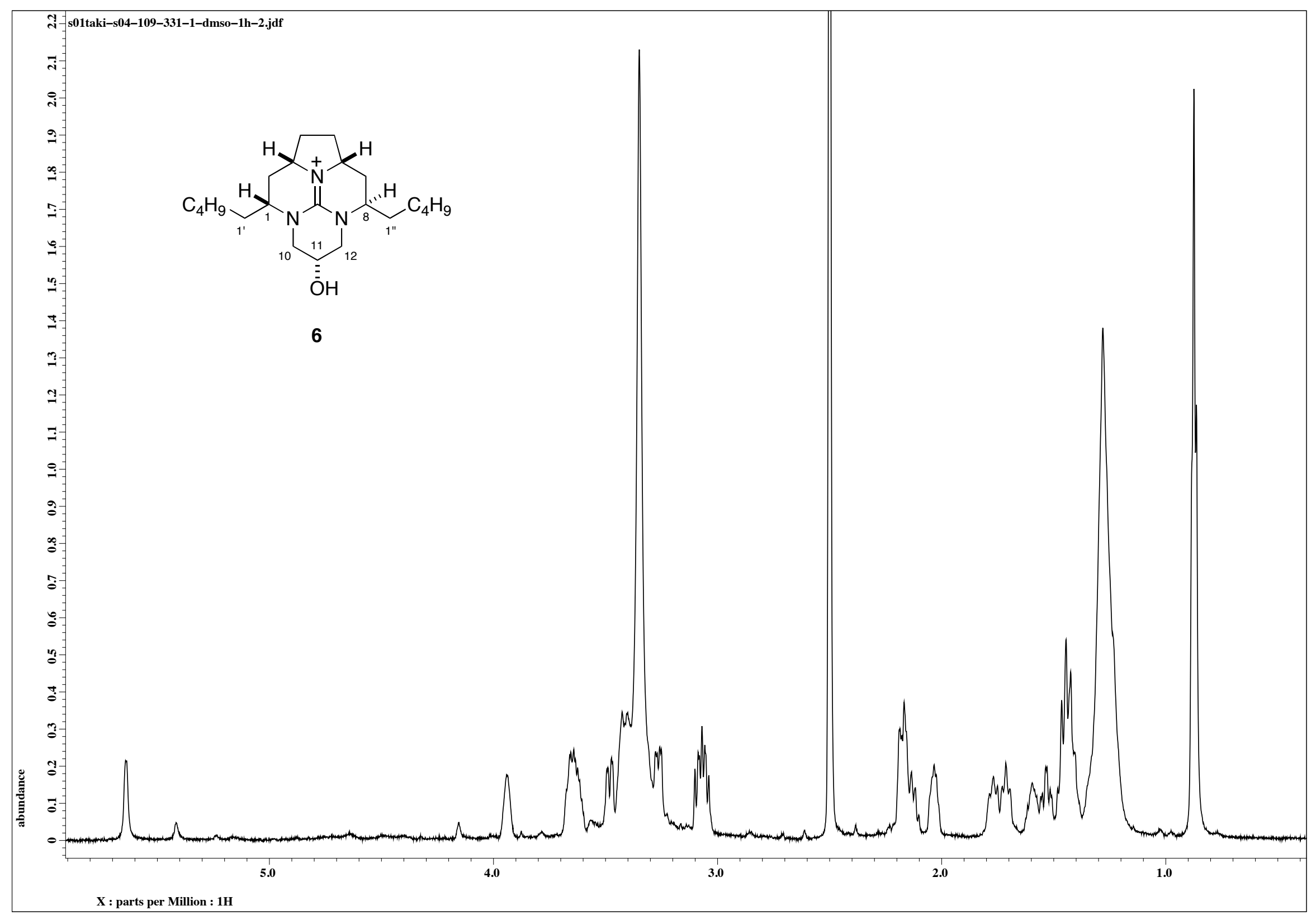

Figure S19. ${ }^{1} \mathrm{H}$ NMR spectrum of 6 in DMSO- $d_{6}$ 


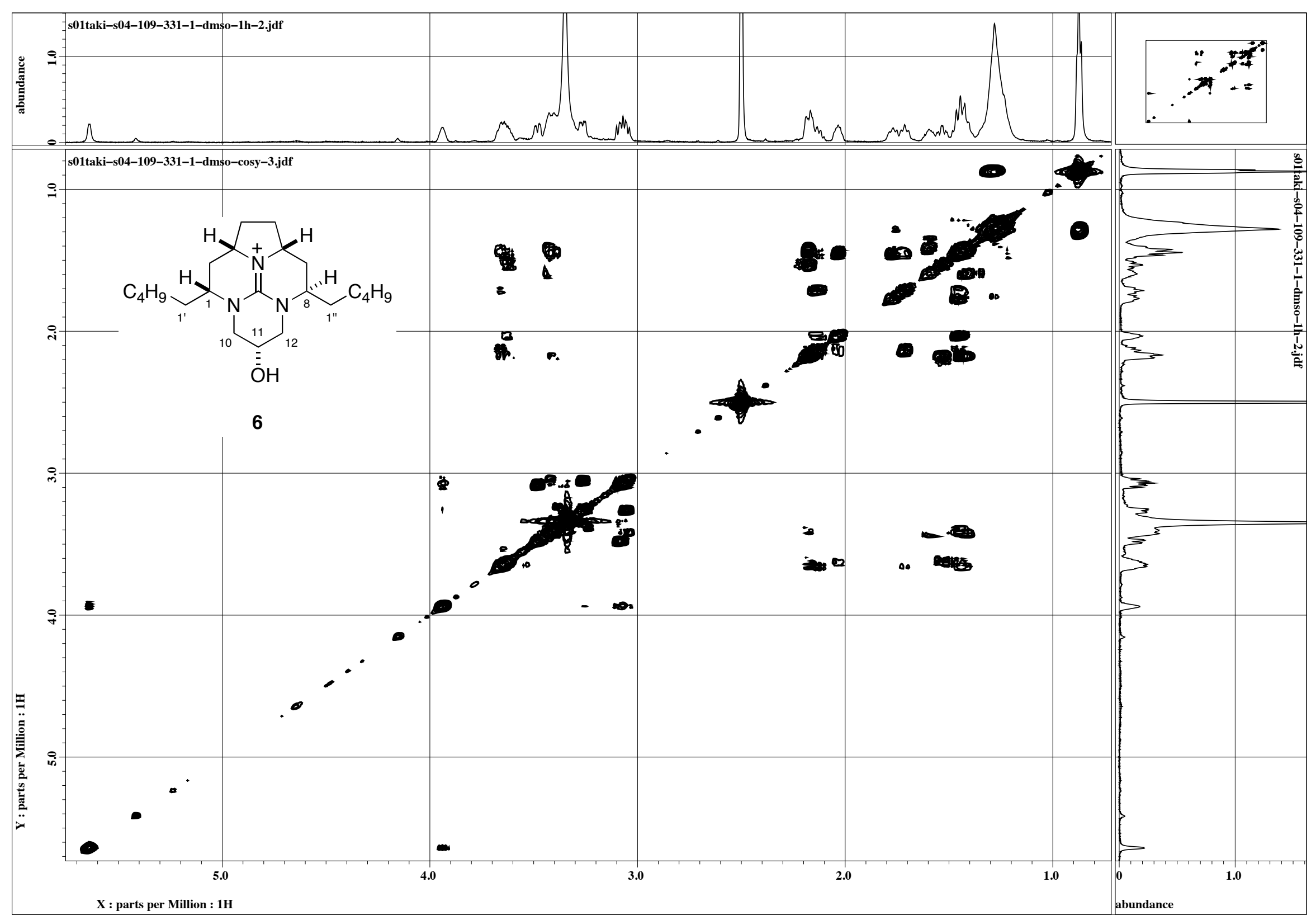

Figure S20. COSY spectrum of 6 in DMSO- $d_{6}$ 


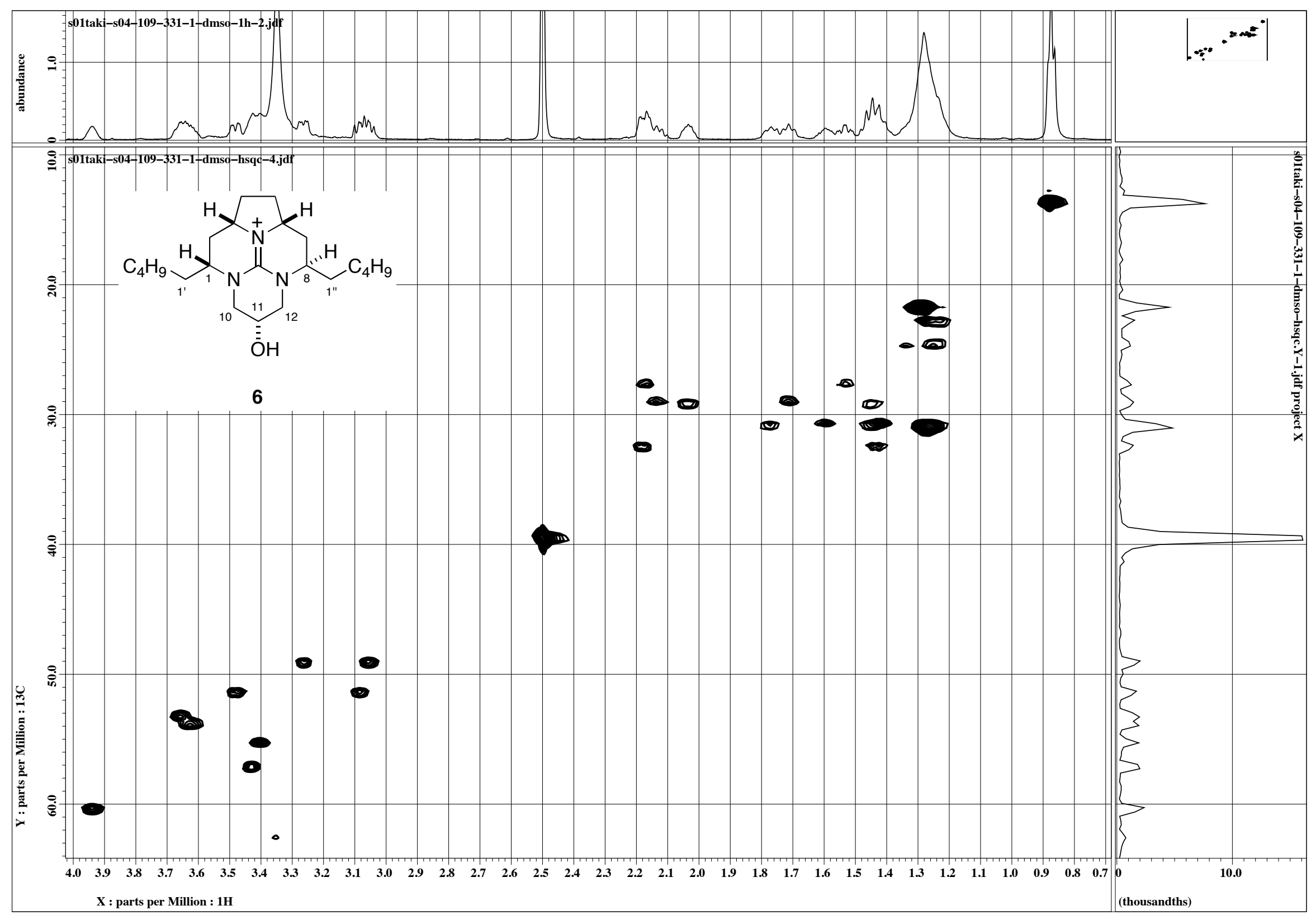

Figure S21. HSQC spectrum of 6 in DMSO- $d_{6}$ 


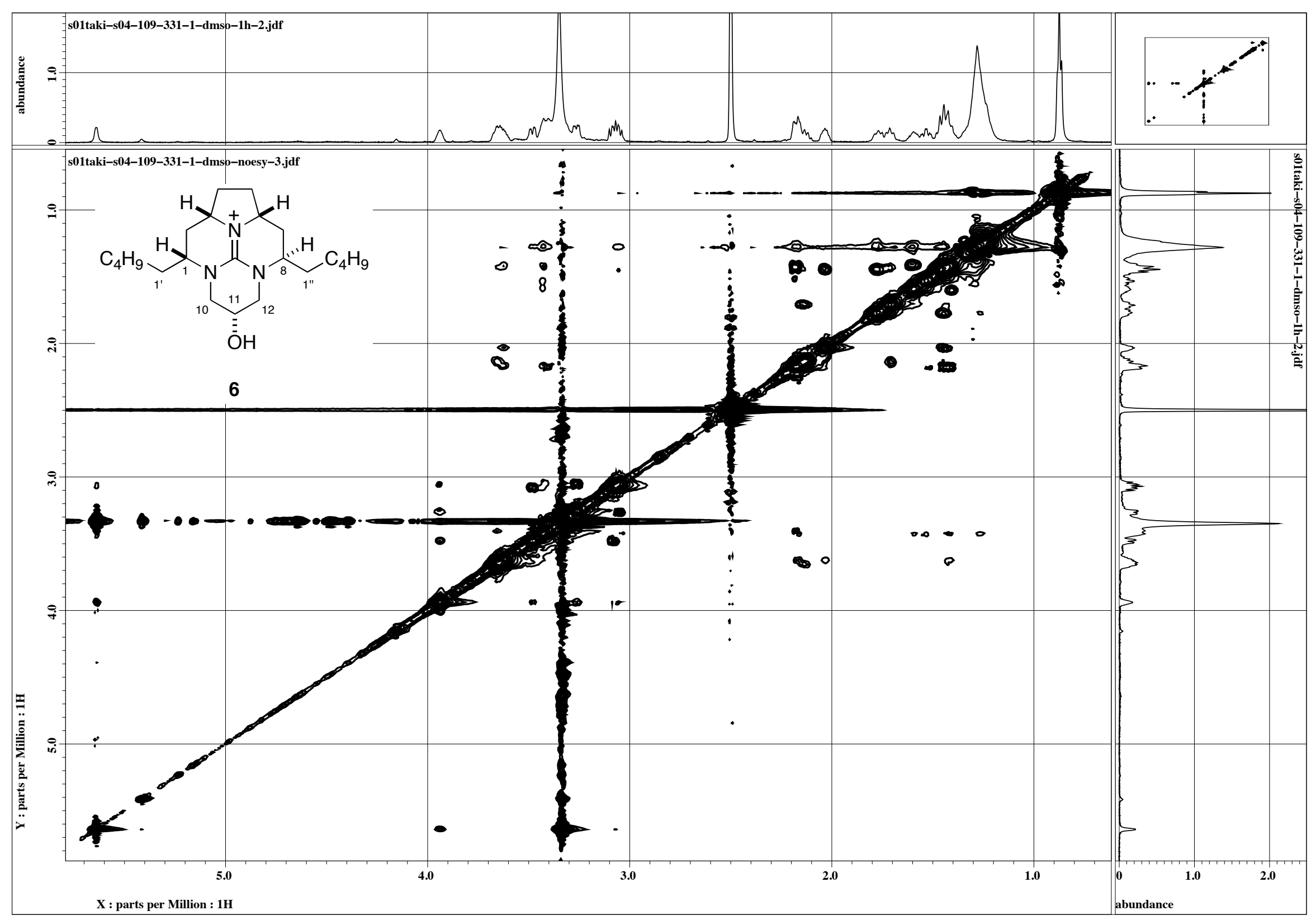

Figure S22. NOESY spectrum of 6 in DMSO- $d_{6}$ 


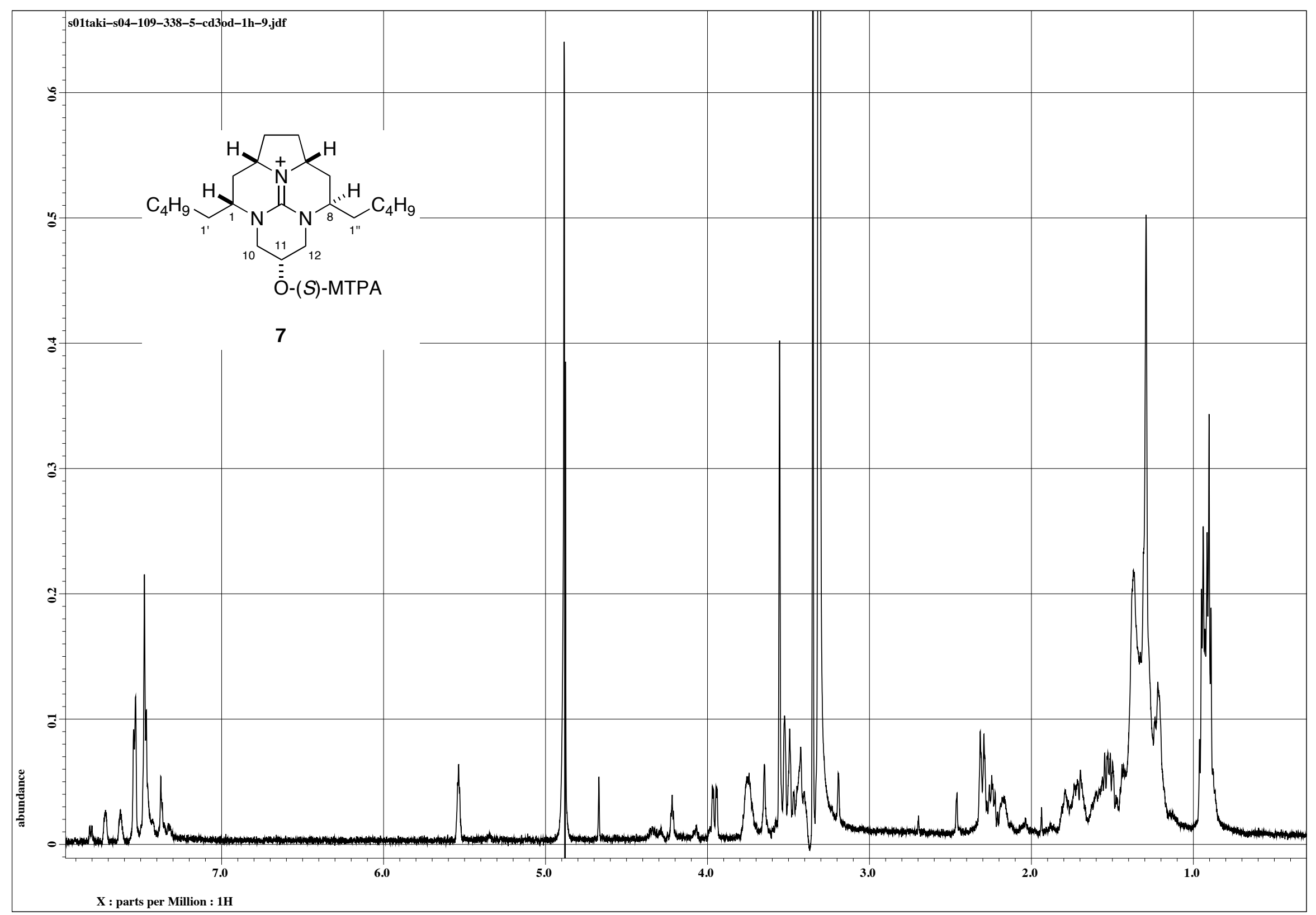

Figure S23. ${ }^{1} \mathrm{H}$ NMR spectrum of 7 in $\mathrm{CD}_{3} \mathrm{OD}$ 


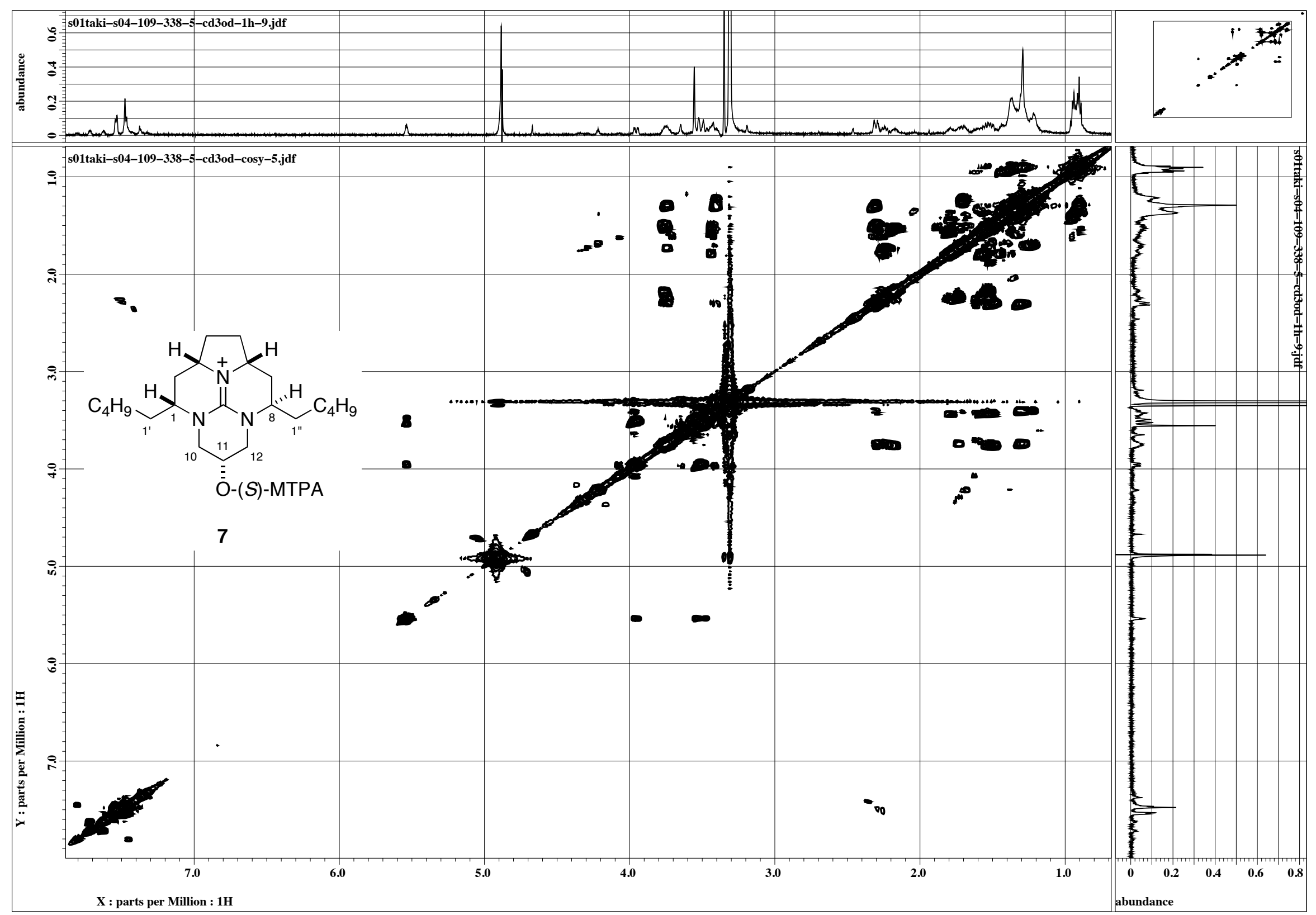

Figure S24. COSY spectrum of 7 in $\mathrm{CD}_{3} \mathrm{OD}$ 


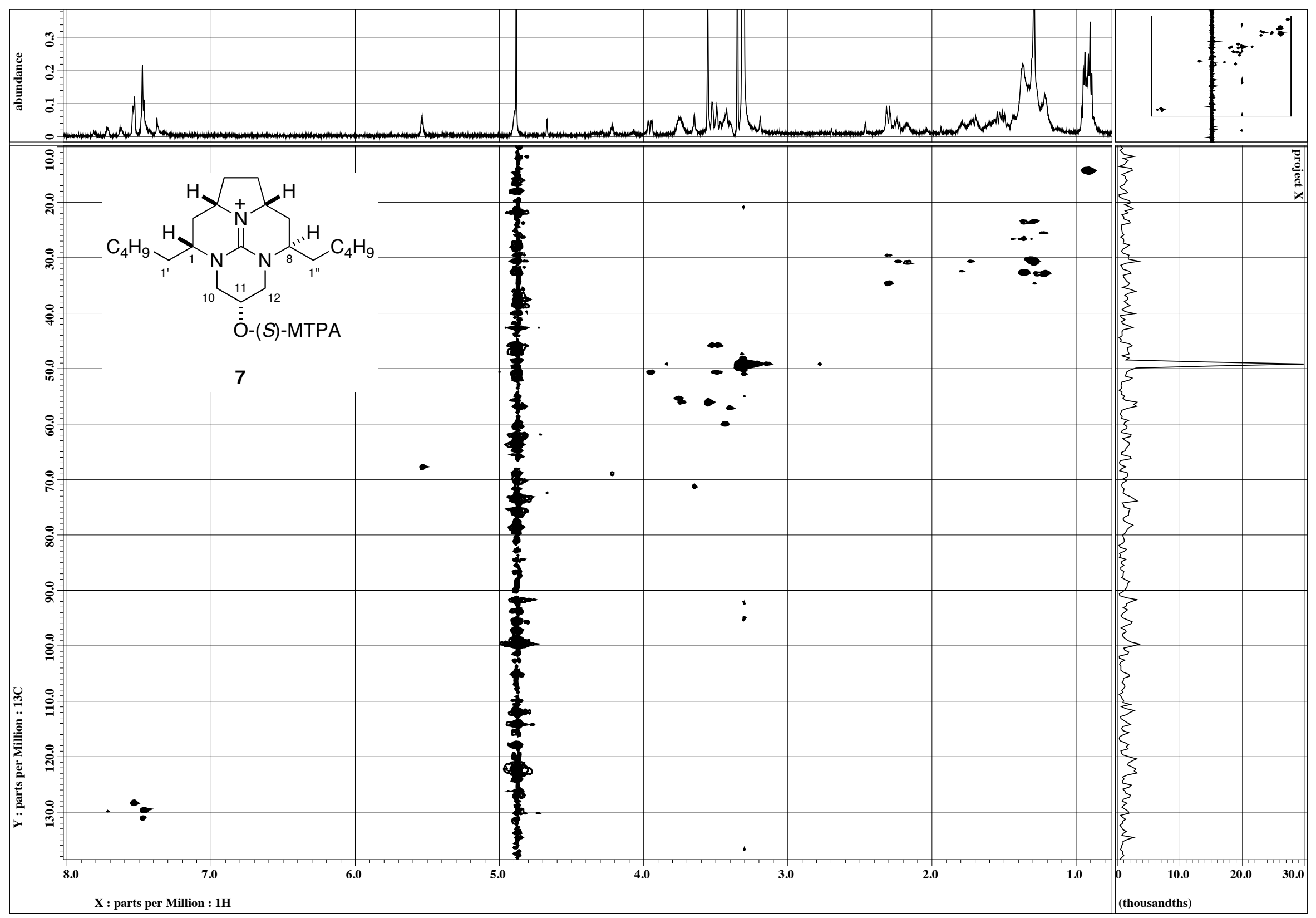

Figure S25. HSQC spectrum of 7 in $\mathrm{CD}_{3} \mathrm{OD}$ 


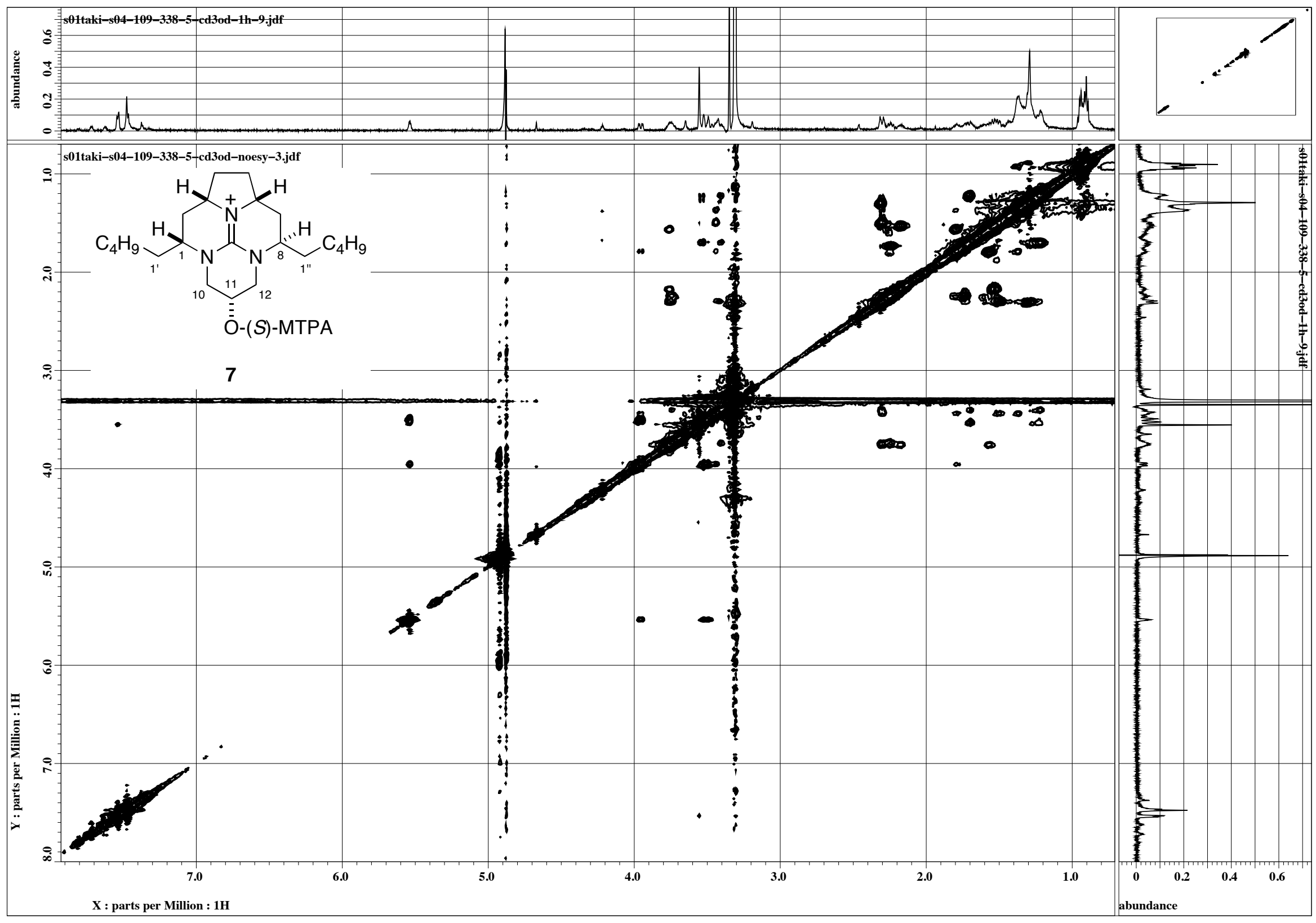

Figure S26. NOESY spectrum of 7 in $\mathrm{CD}_{3} \mathrm{OD}$ 


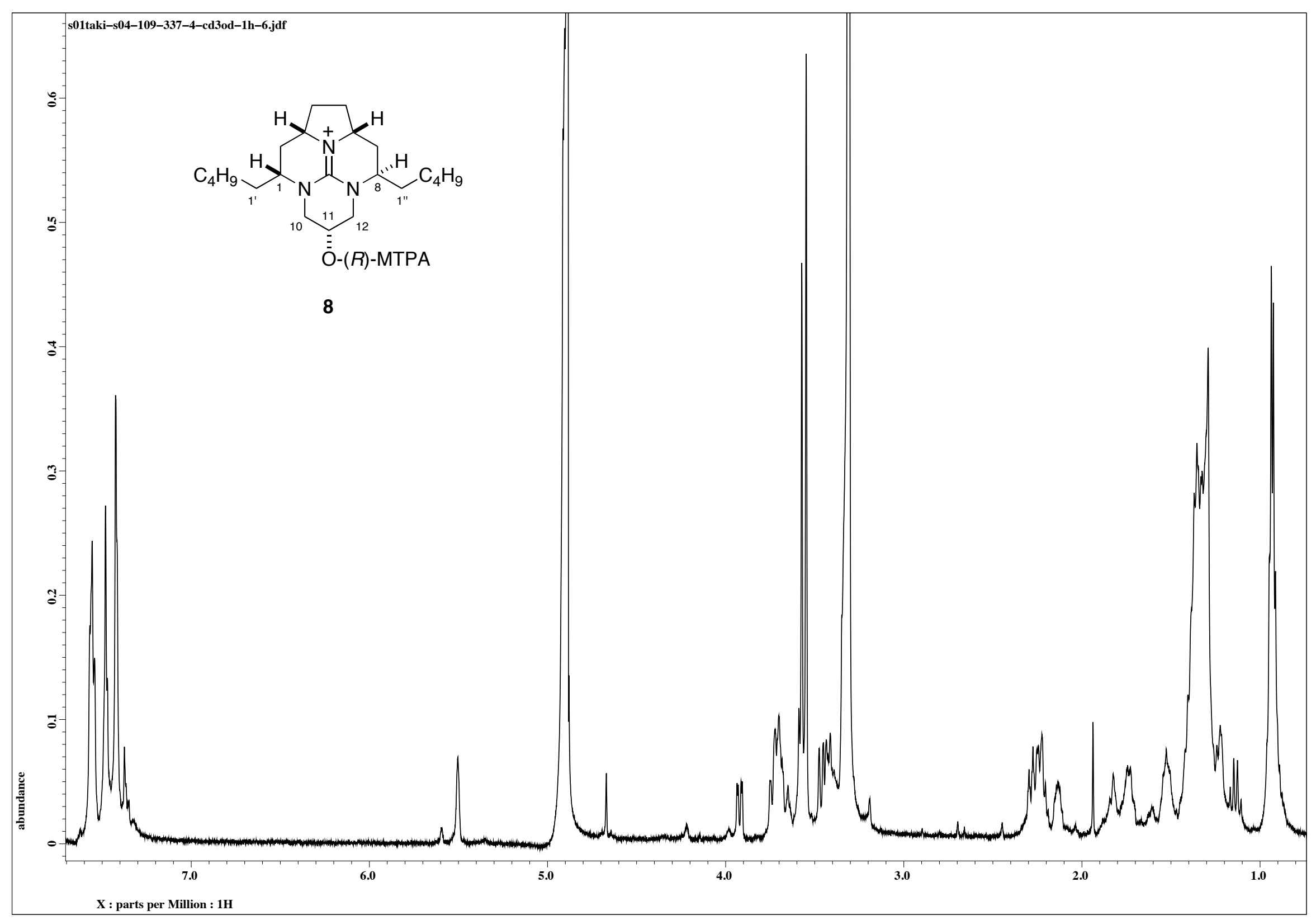

Figure S27. ${ }^{1} \mathrm{H}$ NMR spectrum of $\mathbf{8}$ in $\mathrm{CD}_{3} \mathrm{OD}$ 


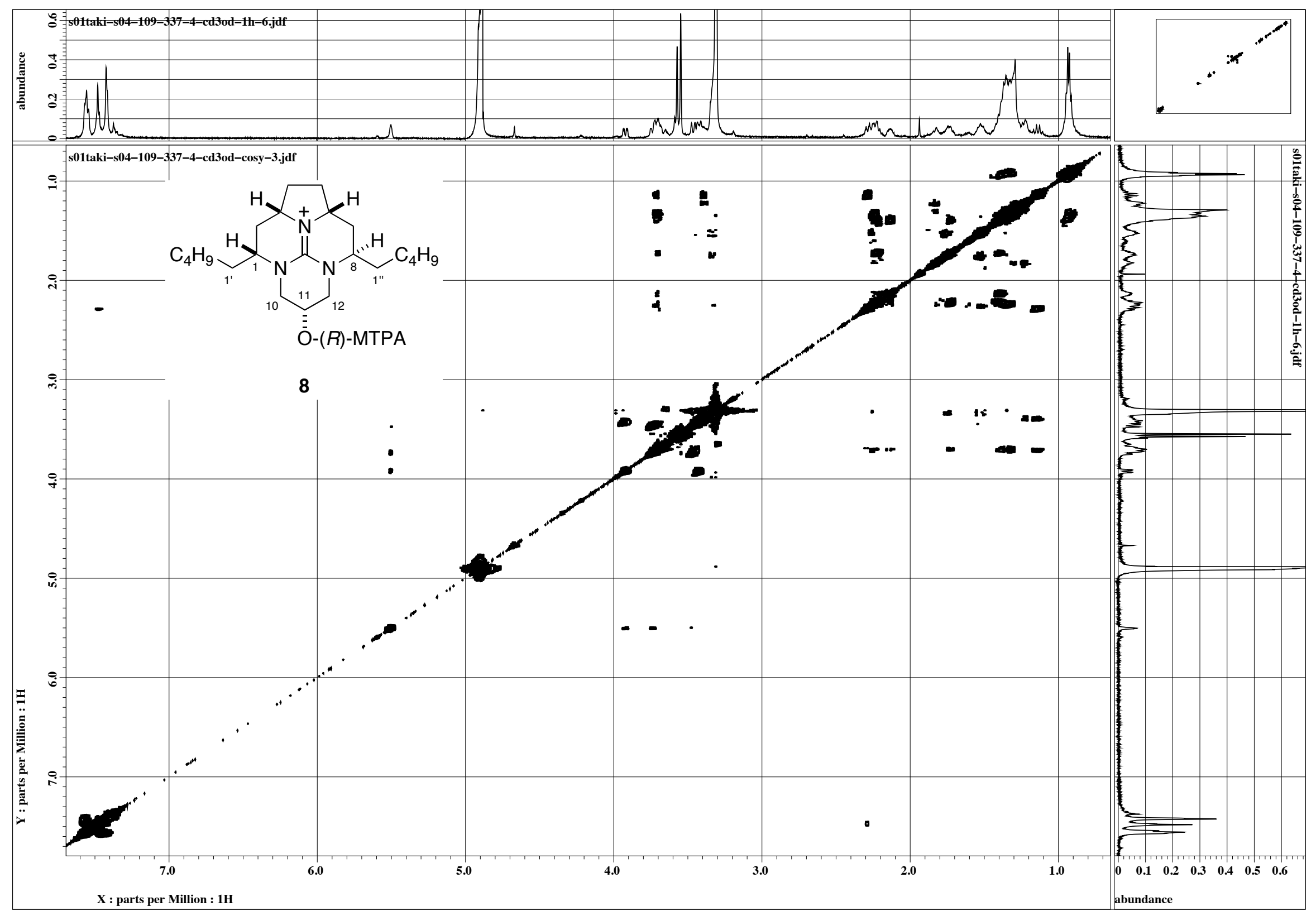

Figure S28. COSY spectrum of $\mathbf{8}$ in $\mathrm{CD}_{3} \mathrm{OD}$ 


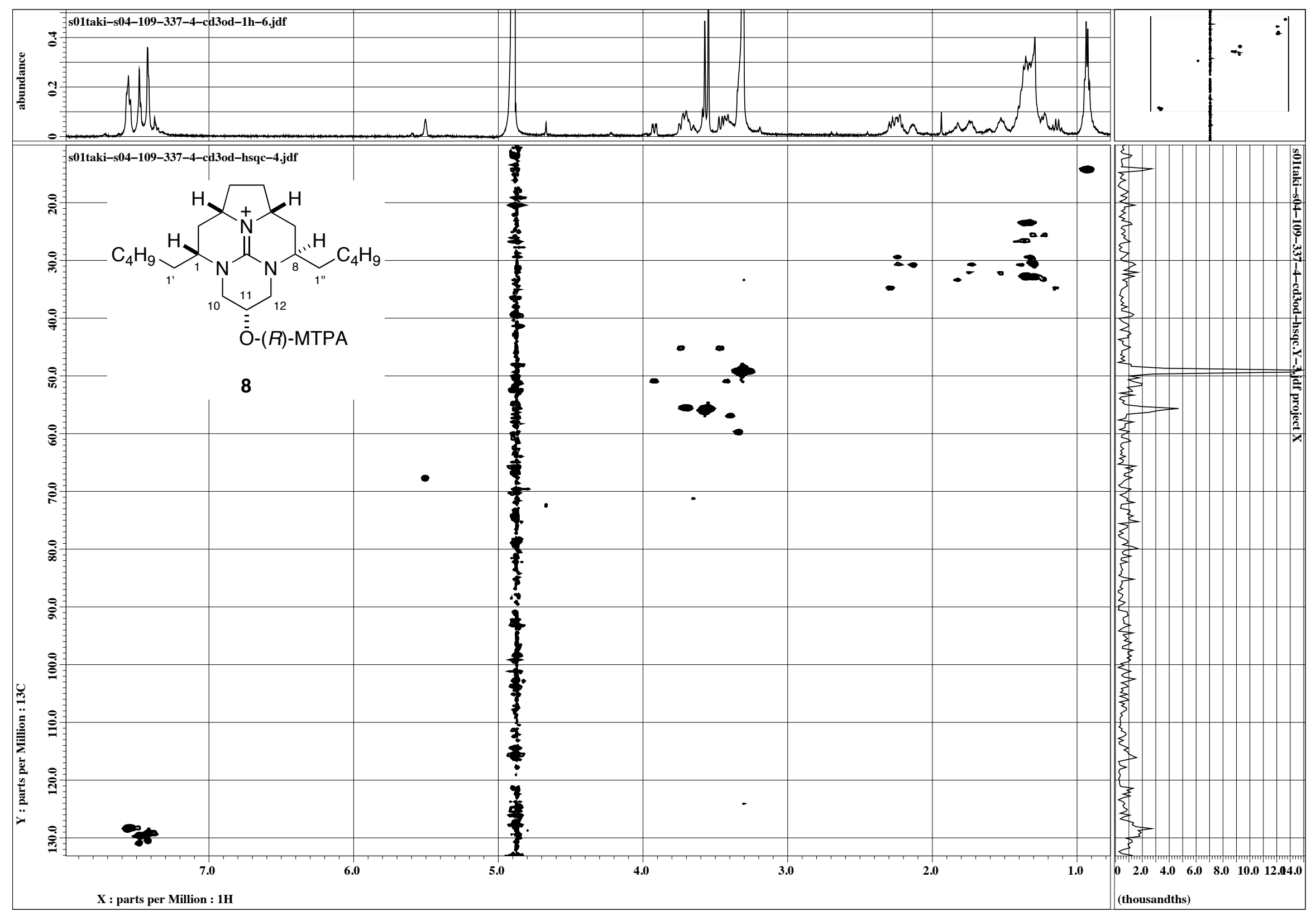

Figure S29. HSQC spectrum of $\mathbf{8}$ in $\mathrm{CD}_{3} \mathrm{OD}$ 


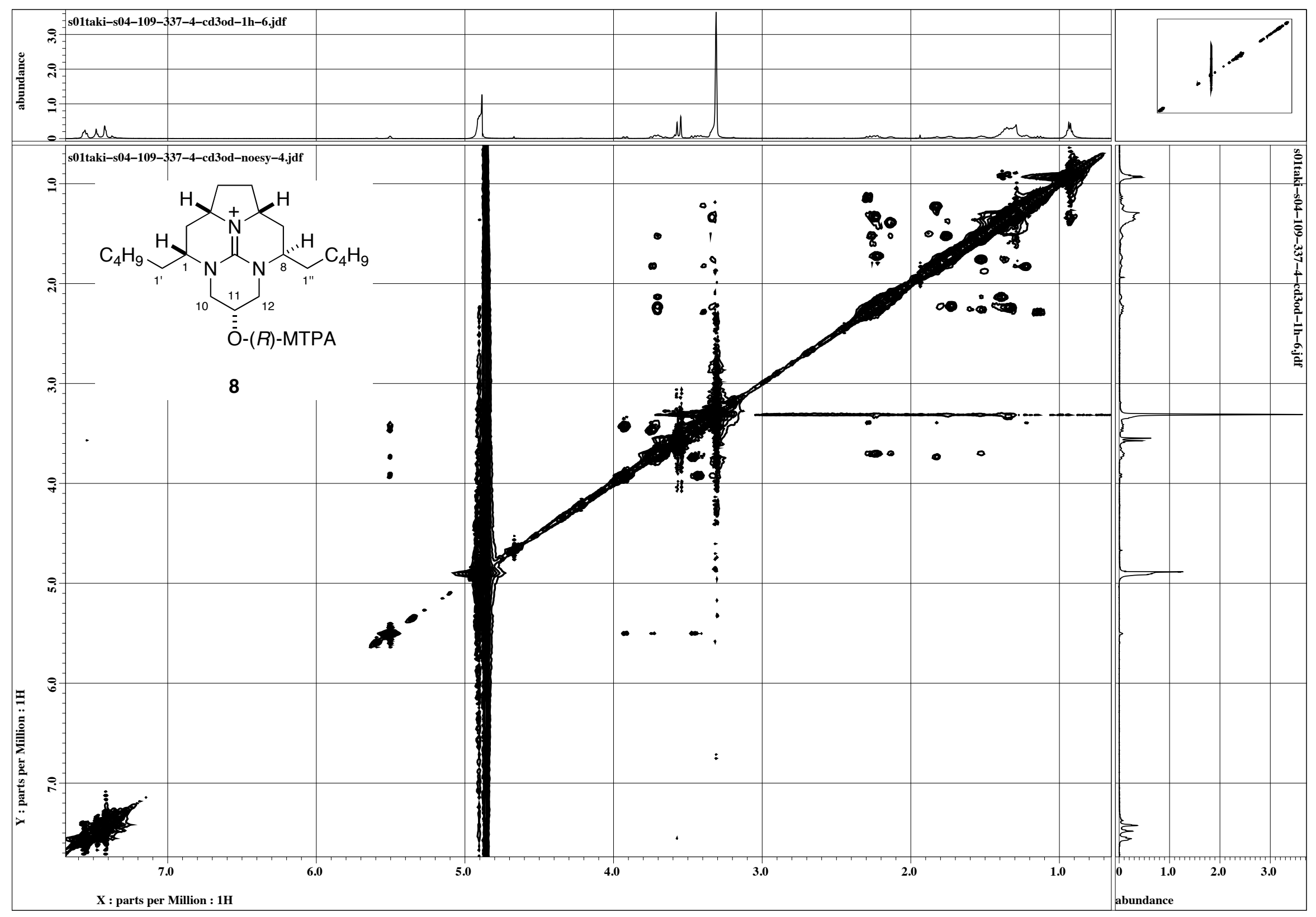

Figure S30. NOESY spectrum of 8 in $\mathrm{CD}_{3} \mathrm{OD}$ 